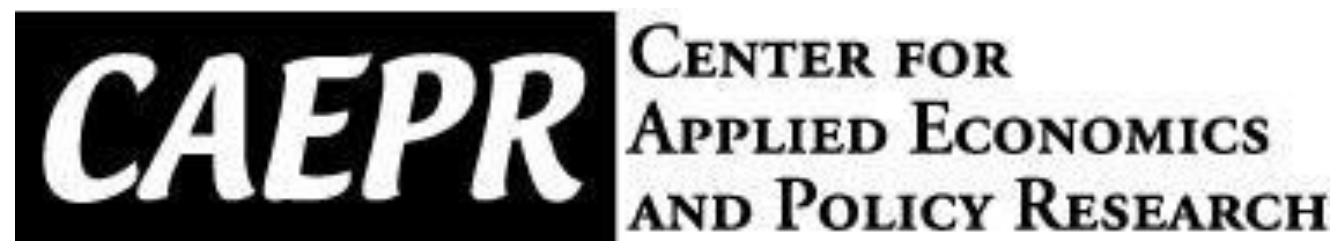

CAEPR Working Paper

\#2014-001

\title{
On the Characterization of Incentive Compatible Mechanisms in General Quasi-linear Environments
}

\author{
Yu Chen \\ Indiana University
}

February 14, 2014

This paper can be downloaded without charge from the Social Science Research Network electronic library at $h$ ttp://ssrn.com/abstract=2395934

The Center for Applied Economics and Policy Research resides in the Department of Economics at Indiana University Bloomington. CAEPR can be found on the Internet at:

http://www.indiana.edu/ caepr. CAEPR can be reached via email at caepr@indiana.edu or via phone at 812-855-4050.

(C)2012 by Yu Chen. All rights reserved. Short sections of text, not to exceed two paragraphs, may be quoted without explicit permission provided that full credit, including $₫$ notice, is given to the source. 


\title{
On the Characterization of Incentive Compatible Mechanisms in General Quasi-linear Environments*
}

\author{
$\mathrm{Yu} \mathrm{Chen}^{\dagger}$
}

\begin{abstract}
This paper generalizes the Myerson (1981)'s characterization results of incentive compatible mechanisms and optimal incentive compatible mechanisms in general quasi-linear multi-agency situations with more abstract payoff function forms and constraint sets. Both Bayesian implementation and ex post implementation are addressed. In this paper the characterization results are also applied to comparison and equivalence between EPIC and BIC mechanisms, strategic dominance of centralized mechanisms over decentralized menus, and optimal (full) surplus extraction.
\end{abstract}

Keywords: Bayesian incentive compatible mechanism, ex post incentive compatible mechanism, quasi-linear environment

JEL Classification: C72 D82 D86

${ }^{*}$ For additional helpful suggestions and comments, I would appreciate Olga Gorelkina, Ran Shao, and Yong Sui. I am solely responsible for any errors.

${ }^{\dagger}$ School of Economics, Nanjing University, 16 Jinyin Street, Anzhong building Rm 2415, Nanjing, Jiangsu 210009, China; (+86)25-83621975, yourchenyu@gmail.com. 


\section{Introduction}

Myerson (1981) in his well-known paper on optimal auction design discusses a similar characterization result concerning Bayesian incentive compatible ${ }^{1}$ mechanisms in a relatively restricted auction setting, which is a special case of the general quasi-linear multi-agency environment. He also extend the characterization result to optimal Bayesian incentive compatible auction mechanisms.

This paper generalizes the characterization results of IC mechanisms ${ }^{2}$ and optimal IC mechanisms in general quasi-linear multi-agency situations. We try to generalize Myerson's characterization results along two directions. 1) we characterize the incentive compatible mechanisms and optimal incentive compatible mechanisms in a more general model setting, especially in more abstract payoff function forms and constraint sets. 2) our characterization results are within not only the context of Bayesian implementation but also that of Ex Post implementation.

Our analysis allows "full interdependence," that is, every agent's payoff depends on all the agents' assignments and types. Although our model is based on a general interdependence situation, all the results still apply to any of its degenerate version, including the separable assignment rules situation and the private valuation situation, in which ex post implementation will degenerate to dominant strategy implementation.

The characterization results of (optimal) IC Mechanisms help us acquire more concrete knowledge about the (optimal) IC Mechanisms and confine them by monotonicity conditions, integration conditions and IR conditions for each agent with the lowest type. Moreover, those results can transfer the original problems to more straightforward or tractable problems and therefore serve as the tools to analyze certain seemingly complicated issues related to optimal mechanism design. In this paper, we also apply the characterization results to comparison and equivalence between EPIC and BIC mechanisms, strategic dominance of centralized mechanisms over decentralized menus, and optimal (full) surplus extraction.

\section{Preliminaries}

We consider a multi-agency contracting game in which one principal (PL) moves first, and $n$ agents, indexed by $i \in \mathcal{N}=\{1, \cdots, n\}$, follow simultaneously and behave non-cooperatively. Agent $i\left(\mathrm{~A}_{i}\right)$ has the private type $\theta_{i} \in \Theta_{i}=\left[\underline{\theta}_{i}, \bar{\theta}_{i}\right]$. We write $\theta=\left(\theta_{i}\right)_{i \in \mathcal{N}} \in \Theta=\prod_{i=1}^{n} \Theta_{i}$ and $\theta_{-i}=$ $\left(\theta_{j}\right)_{j \in \mathcal{N} \backslash\{i\}} \in \theta_{-i}=\prod_{j \neq i}^{n} \theta_{j} . \theta$ has a joint distribution function $F: \Theta \rightarrow[0,1]$ and an associated joint density function $f: \Theta \rightarrow \mathbb{R}_{+}$with respect to Lebesgue measure. For each $i \in \mathcal{N}$, there are a marginal distribution function $F_{i}: \Theta_{i} \rightarrow[0,1]$ and an associated conditional distribution function of $\theta_{-i}$ on $\theta_{i}, F_{-i}\left(\cdot \mid \theta_{i}\right): \Theta_{-i} \rightarrow[0,1] . F_{i}$ has a marginal density function with respect to

\footnotetext{
${ }^{1}$ Actually there is no Bayesian update belief, since Myerson assumes independent types.

${ }^{2}$ Due to the revelation principle, we can restrict our attention to incentive compatible (direct) mechanisms out of general mechanisms in mechanism design.
} 
Lebesgue measure, $f_{i}: \Theta_{i} \rightarrow \mathbb{R}_{+}$, and $F_{-i}\left(\cdot \mid \theta_{i}\right)$ has a conditional density function with respect to Lebesgue measure, $f_{-i}\left(\cdot \mid \theta_{i}\right): \Theta_{-i} \rightarrow \mathbb{R}_{+}$. In addition, $f(\theta)=f_{-i}\left(\theta_{-i} \mid \theta_{i}\right) f_{i}\left(\theta_{i}\right)$,for all $\theta \in \Theta$.

Contracts consist of two parts: assignments (or say activities, decision rules, alternatives, etc.) and transfers. The end-of-period commonly observable assignments for the agents is $x \in \mathcal{X}$, where $\mathcal{X}$ is a partial-ordered set of a metric space. The end-of-period commonly observable monetary transfer from PL to $A_{i}$ is $t_{i} \in \mathbb{R}$. Let Borel measurable function $\mathbf{x}: \Theta \rightarrow \mathcal{X}$ denote assignment rule, which specifies to the agents the assignments for each type report profile of the agents. Let Borel measurable function $\mathbf{t}_{i}: \Theta \rightarrow \mathbb{R}$ denote transfer rule, which specifies to $\mathrm{A}_{i}$ a transfer for each type report profile of the agents ${ }^{3}$. We write $\mathbf{t}=\prod_{i=1}^{n} \mathbf{t}_{i}$. A mechanism a pair of functions $(\mathbf{x}, \mathbf{t})$ satisfying $\mathbf{x}(\theta) \in X \subseteq \mathcal{X}$ and $\mathbf{t}(\theta) \in T \subseteq \mathbb{R}^{n}$. A certain feasible constraint on contracting is embodied in $X$ and $T$ as the subsets of $\mathcal{X}$ and $\mathbb{R}^{n}$.

We focus on the classic quasi-linear payoffs. PL's ex post payoff function $U: \mathcal{X} \times \mathbb{R}^{n} \times \Theta \rightarrow \mathbb{R}$ takes the quasi-linear form:

$$
U(x, t, \theta) \equiv u(x, \theta)-\sum_{i=1}^{n} t_{i},
$$

where $u: \mathcal{X} \times \Theta \rightarrow \mathbb{R}$ is the payoff function from the agents' assignments. $\mathrm{A}_{i}$ 's ex post payoff function $V_{i}: \mathcal{X} \times \mathbb{R} \times \Theta \rightarrow \mathbb{R}$ takes the quasi-linear form:

$$
V_{i}\left(x, t_{i}, \theta\right) \equiv v_{i}(x, \theta)+t_{i}
$$

where $v_{i}: \mathcal{X} \times \Theta \rightarrow \mathbb{R}$ is the payoff function from the agents' assignments. $v_{i}$ is continuous. $\mathrm{A}_{i}$ has the reservation utility $r_{i} \in \mathbb{R}$.

\section{Principal-Agent Problems over Mechanism Design}

Under the general quasi-linear environment, there are two major classes of implementation are notable for study, depending on the information structure. The first class is Bayesian implementation in terms of Bayesian incentive compatible mechanism.

A direct mechanism $(\mathbf{x}, \mathbf{t})$ is Bayesian incentive compatible (BIC) if for all $i \in \mathcal{N}$ and $\theta_{i} \in \Theta_{i}$

$$
\int_{\Theta_{-i}} v_{i}(\mathbf{x}(\theta), \theta)+\mathbf{t}_{i}(\theta) d F_{-i}\left(\theta_{-i} \mid \theta_{i}\right) \geq \int_{\Theta_{-i}} v_{i}\left(\mathbf{x}\left(\theta_{i}^{\prime}, \theta_{-i}\right), \theta\right)+\mathbf{t}_{i}\left(\theta_{i}^{\prime}, \theta_{-i}\right) d F_{-i}\left(\theta_{-i} \mid \theta_{i}\right),
$$

for all $\theta_{i}^{\prime} \in \Theta_{i}$. A BIC mechanism (x,t) is (interim) Individual Rational (IR) if for all $i \in \mathcal{N}$ and $\theta_{i} \in \Theta_{i}$,

$$
\int_{\Theta_{-i}} v_{i}(\mathbf{x}(\theta), \theta)+\mathbf{t}_{i}(\theta) d F_{-i}\left(\theta_{-i} \mid \theta_{i}\right) \geq r_{i}
$$

\footnotetext{
${ }^{3}$ Note that $\mathbf{t}_{i}$ must be Lebesgue measurable as well.
} 
We thus present Principal-Agent problem with Bayesian implementation.

(P1) $\max _{(\mathbf{x}, \mathbf{t})} \int_{\Theta} u(\mathbf{x}(\theta), \theta)-\sum_{i=1}^{n} \mathbf{t}_{i}(\theta) d F(\theta)$

s.t. $(\mathbf{x}, \mathbf{t})$ is $\mathbf{B I C}$ and $\mathbf{I R}$

For all $\theta \in \Theta, \mathbf{x}(\theta) \in X, \mathbf{t}(\theta) \in T$

The second class is ex post implementation in terms of ex post incentive compatible mechanism. A direct mechanism (x, t) is Ex Post incentive compatible (EPIC) if for all $i \in \mathcal{N}$ and $\theta \in \Theta$,

$$
v_{i}(\mathbf{x}(\theta), \theta)+\mathbf{t}_{i}(\theta) \geq v_{i}\left(\mathbf{x}\left(\theta_{i}^{\prime}, \theta_{-i}\right), \theta\right)+\mathbf{t}_{i}\left(\theta_{i}^{\prime}, \theta_{-i}\right),
$$

for all $\theta_{i}^{\prime} \in \Theta_{i}$. A EPIC mechanism (x,t) is (ex post) Individual Rational (IR) if for all $i \in \mathcal{N}$ and $\theta \in \Theta$,

$$
v_{i}(\mathbf{x}(\theta), \theta)+\mathbf{t}_{i}(\theta) \geq r_{i}
$$

We thus present Principal-Agent problem with Bayesian implementation.

(P2) $\max _{(\mathbf{x}, \mathbf{t})} \int_{\Theta} u(\mathbf{x}(\theta), \theta)-\sum_{i=1}^{n} \mathbf{t}_{i}(\theta) d F(\theta)$

s.t. $(\mathbf{x}, \mathbf{t})$ is EPIC and IR

For all $\theta \in \Theta, \mathbf{x}(\theta) \in X, \mathbf{t}(\theta) \in T$

We also need some assumptions for our analysis as below.

Assumption $1 v_{i}(x, \theta)$ is Borel-measurable in $x^{4}$ and differentiable in $\theta_{i} \in\left[\underline{\theta}_{i}, \bar{\theta}_{i}\right]$.

Assumption $2 \frac{\partial v_{i}(x, \theta)}{\partial \theta_{i}}$ is continuous in $x$ and $\theta$.

Assumption $3 u(x, \theta)$ is Borel measurable in $x$ and $\theta .{ }^{5}$

Assumption $4 v_{i}(x, \theta)$ has the strictly increasing difference (stronger than single crossing property) in $x$ and $\theta_{i}$, for all $\theta_{-i} \in \Theta_{-i}$, that is,

$\forall x^{\prime \prime}>x^{\prime}$ in $\mathcal{X}, b_{i}>a_{i}$ in $\Theta_{i}$,

$$
v_{i}\left(x^{\prime \prime}, b_{i}, \theta_{-i}\right)-v_{i}\left(x^{\prime}, b_{i}, \theta_{-i}\right)>v_{i}\left(x^{\prime \prime}, a_{i}, \theta_{-i}\right)-v_{i}\left(x^{\prime}, a_{i}, \theta_{-i}\right)
$$

Or equivalently, $\frac{\partial v_{i}(x, \theta)}{\partial \theta_{i}}$ is strictly increasing in $x$.

Moreover, we define

$$
\bar{V}_{i}\left(\theta ; \mathbf{x}, \mathbf{t}_{i}\right)=v_{i}(\mathbf{x}(\theta), \theta)+\mathbf{t}_{i}(\theta)
$$

as $\mathrm{A}_{i}$ 's ex post equilibrium payoff with respect to direct mechanism $(\mathbf{x}, \mathbf{t})$ when he truthfully reports his type $\theta_{i}$ given other agents' truthful type reports $\theta_{-i}$. We also define

$$
\widehat{V}_{i}\left(\theta_{i} ; \mathbf{x}, \mathbf{t}_{i}\right)=\int_{\Theta_{-i}} \bar{V}_{i}\left(\theta ; \mathbf{x}, \mathbf{t}_{i}\right) d F_{-i}\left(\theta_{-i} \mid \theta_{i}\right)=\int_{\Theta_{-i}} v_{i}(\mathbf{x}(\theta), \theta)+\mathbf{t}_{i}(\theta) d F_{-i}\left(\theta_{-i} \mid \theta_{i}\right)
$$

as $\mathrm{A}_{i}$ 's interim (Bayesian) equilibrium payoff with respect to direct mechanism $(\mathbf{x}, \mathbf{t})$ when he truthfully reports his type $\theta_{i}$.

\footnotetext{
${ }^{4}$ To be more specific, we can assume $v_{i}(x, \theta)$ is continuous in $x$.

${ }^{5}$ To be more specific, we can assume $u(x, \theta)$ is continuous in $x$ and $\theta$.
} 


\section{Characterization Results of IC Mechanisms}

\subsection{Characterization of BIC Mechanisms}

It is difficult to generalize Myerson's Characterization result in a general Bayesian information structure. We instead address a particular but still important case of information structure.

Definition 1 There are no substantive Bayesian update beliefs in the information structure of mechanism design if in the information structure on which $P L$ and the agents rely, $F_{-i}\left(\theta_{-i} \mid \theta_{i}\right) \equiv$ $F_{-i}\left(\theta_{-i} \mid \theta_{i}^{\prime}\right) \equiv F_{-i}\left(\theta_{-i}\right), \forall \theta_{-i}, \theta_{i}, \theta_{i}^{\prime}$. Moreover, $f_{-i}\left(\theta_{-i} \mid \theta_{i}\right) \equiv f_{-i}\left(\theta_{-i} \mid \theta_{i}^{\prime}\right) \equiv f_{-i}\left(\theta_{-i}\right), \forall \theta_{-i}, \theta_{i}, \theta_{i}^{\prime}$.

If $\theta$ is independently distributed, then $\theta_{i}$ has a distribution function $F_{i}: \Theta_{i} \rightarrow[0,1]$ and an associated density function with respect to Lebesgue measure $f_{i}: \Theta_{i} \rightarrow \mathbb{R}_{+}$. There must not be substantive Bayesian update beliefs in the information structure. In this case, we can provide a generalized result of characterization of BIC mechanisms with more straightforward and economically intuitive conditions.

Proposition 1 Suppose that there are no substantive Bayesian update beliefs. Under assumptions 1 and 2, a direct mechanism (x,t) is BIC and IR iff for each $i \in \mathcal{N}$,

$$
\begin{aligned}
& \int_{\Theta_{-i}} \frac{\partial v_{i}\left(\mathbf{x}\left(h, \theta_{-i}\right), \theta\right)}{\partial \theta_{i}} d F_{-i}\left(\theta_{-i}\right) \text { is increasing in } h \text { on } \Theta_{i}, \\
\widehat{V}_{i}\left(b_{i} ; \mathbf{x}, \mathbf{t}_{i}\right)-\widehat{V}_{i}\left(a_{i} ; \mathbf{x}, \mathbf{t}_{i}\right)= & \int_{a_{i}}^{b_{i}} \int_{\Theta_{-i}}\left[\frac{\partial v_{i}(\mathbf{x}(\theta), \theta)}{\partial \theta_{i}}\right] d F_{-i}\left(\theta_{-i}\right) d \theta_{i}, \forall a_{i}, b_{i} \in \Theta_{i},{ }^{6} \\
\widehat{V}_{i}\left(\underline{\theta}_{i} ; \mathbf{x}, \mathbf{t}_{i}\right) \geq & r_{i} .
\end{aligned}
$$

Proof. " $\Longrightarrow "$ BIC condition implies that $\forall a_{i}<b_{i} \in \Theta_{i}$,

$$
\begin{aligned}
\widehat{V}_{i}\left(b_{i} ; \mathbf{x}, \mathbf{t}_{i}\right) \geq & \int_{\Theta_{-i}} v_{i}\left(\mathbf{x}\left(a_{i}, \theta_{-i}\right), b_{i}, \theta_{-i}\right)+\mathbf{t}_{i}\left(a_{i}, \theta_{-i}\right) d F_{-i}\left(\theta_{-i}\right) \\
= & \int_{\Theta_{-i}} v_{i}\left(\mathbf{x}\left(a_{i}, \theta_{-i}\right), a_{i}, \theta_{-i}\right)+\mathbf{t}_{i}\left(a_{i}, \theta_{-i}\right) d F_{-i}\left(\theta_{-i}\right) \\
& +\int_{\Theta_{-i}} v_{i}\left(\mathbf{x}\left(a_{i}, \theta_{-i}\right), b_{i}, \theta_{-i}\right) d F_{-i}\left(\theta_{-i}\right)-\int_{\Theta_{-i}} v_{i}\left(\mathbf{x}\left(a_{i}, \theta_{-i}\right), a_{i}, \theta_{-i}\right) d F_{-i}\left(\theta_{-i}\right) \\
= & \widehat{V}_{i}\left(a_{i} ; \mathbf{x}, \mathbf{t}_{i}\right) \\
& +\int_{\Theta_{-i}} v_{i}\left(\mathbf{x}\left(a_{i}, \theta_{-i}\right), b_{i}, \theta_{-i}\right) d F_{-i}\left(\theta_{-i}\right)-\int_{\Theta_{-i}} v_{i}\left(\mathbf{x}\left(a_{i}, \theta_{-i}\right), a_{i}, \theta_{-i}\right) d F_{-i}\left(\theta_{-i}\right)
\end{aligned}
$$

Thus,

$$
\begin{aligned}
& \widehat{V}_{i}\left(b_{i} ; \mathbf{x}, \mathbf{t}_{i}\right)-\widehat{V}_{i}\left(a_{i} ; \mathbf{x}, \mathbf{t}_{i}\right) \\
\geq & \int_{\Theta_{-i}} v_{i}\left(\mathbf{x}\left(a_{i}, \theta_{-i}\right), b_{i}, \theta_{-i}\right) d F_{-i}\left(\theta_{-i}\right)-\int_{\Theta_{-i}} v_{i}\left(\mathbf{x}\left(a_{i}, \theta_{-i}\right), a_{i}, \theta_{-i}\right) d F_{-i}\left(\theta_{-i}\right)
\end{aligned}
$$


Reversing $b_{i}$ and $a_{i}$ in (4) and combining with (5), we have

$$
\begin{aligned}
& \int_{\Theta_{-i}} v_{i}\left(\mathbf{x}\left(a_{i}, \theta_{-i}\right), b_{i}, \theta_{-i}\right) d F_{-i}\left(\theta_{-i}\right)-\int_{\Theta_{-i}} v_{i}\left(\mathbf{x}\left(a_{i}, \theta_{-i}\right), a_{i}, \theta_{-i}\right) d F_{-i}\left(\theta_{-i}\right) \\
\leq & \widehat{V}_{i}\left(b_{i} ; \mathbf{x}, \mathbf{t}_{i}\right)-\widehat{V}_{i}\left(a_{i} ; \mathbf{x}, \mathbf{t}_{i}\right) \\
\leq & \int_{\Theta_{-i}} v_{i}\left(\mathbf{x}\left(b_{i}, \theta_{-i}\right), b_{i}, \theta_{-i}\right) d F_{-i}\left(\theta_{-i}\right)-\int_{\Theta_{-i}} v_{i}\left(\mathbf{x}\left(b_{i}, \theta_{-i}\right), a_{i}, \theta_{-i}\right) d F_{-i}\left(\theta_{-i}\right)
\end{aligned}
$$

Hence,

$$
\begin{aligned}
& \int_{\Theta_{-i}} v_{i}\left(\mathbf{x}\left(a_{i}, \theta_{-i}\right), b_{i}, \theta_{-i}\right) d F_{-i}\left(\theta_{-i}\right)-\int_{\Theta_{-i}} v_{i}\left(\mathbf{x}\left(a_{i}, \theta_{-i}\right), a_{i}, \theta_{-i}\right) d F_{-i}\left(\theta_{-i}\right) \\
\leq & \int_{\Theta_{-i}} v_{i}\left(\mathbf{x}\left(b_{i}, \theta_{-i}\right), b_{i}, \theta_{-i}\right) d F_{-i}\left(\theta_{-i}\right)-\int_{\Theta_{-i}} v_{i}\left(\mathbf{x}\left(b_{i}, \theta_{-i}\right), a_{i}, \theta_{-i}\right) d F_{-i}\left(\theta_{-i}\right)
\end{aligned}
$$

Now suppose (1) does not hold, then for some $b_{i}^{\prime}>a_{i}^{\prime}$,

$$
\int_{\Theta_{-i}} \frac{\partial v_{i}\left(\mathbf{x}\left(b_{i}^{\prime}, \theta\right), \theta_{i}, \theta_{-i}\right)}{\partial \theta_{i}} d F_{-i}\left(\theta_{-i}\right)<\int_{\Theta_{-i}} \frac{\partial v_{i}\left(\mathbf{x}\left(a_{i}^{\prime}, \theta\right), \theta_{i}, \theta_{-i}\right)}{\partial \theta_{i}} d F_{-i}\left(\theta_{-i}\right)
$$

Thus,

$$
\begin{aligned}
\int_{a_{i}^{\prime}}^{b_{i}^{\prime}} \int_{\Theta_{-i}} \frac{\partial v_{i}\left(\mathbf{x}\left(b_{i}^{\prime}, \theta\right), \theta_{i}, \theta_{-i}\right)}{\partial \theta_{i}} d F_{-i}\left(\theta_{-i}\right) d \theta_{i} & <\int_{a_{i}^{\prime}}^{b_{i}^{\prime}} \int_{\Theta_{-i}} \frac{\partial v_{i}\left(\mathbf{x}\left(a_{i}^{\prime}, \theta\right), \theta_{i}, \theta_{-i}\right)}{\partial \theta_{i}} d F_{-i}\left(\theta_{-i}\right) d \theta_{i} \\
\int_{\Theta_{-i}} \int_{a_{i}}^{b_{i}^{\prime}} \frac{\partial v_{i}\left(\mathbf{x}\left(b_{i}^{\prime}, \theta\right), \theta_{i}, \theta_{-i}\right)}{\partial \theta_{i}} d \theta_{i} d F_{-i}\left(\theta_{-i}\right) & <\int_{\Theta_{-i}} \int_{a_{i}^{\prime}}^{b_{i}^{\prime}} \frac{\partial v_{i}\left(\mathbf{x}\left(a_{i}^{\prime}, \theta\right), \theta_{i}, \theta_{-i}\right)}{\partial \theta_{i}} d \theta_{i} d F_{-i}\left(\theta_{-i}\right) \\
\int_{\Theta_{-i}} v_{i}\left(\mathbf{x}\left(b_{i}^{\prime}, \theta_{-i}\right), b_{i}^{\prime}, \theta_{-i}\right)-v_{i}\left(\mathbf{x}\left(b_{i}^{\prime}, \theta_{-i}\right), a_{i}^{\prime}, \theta_{-i}\right) d F_{-i}\left(\theta_{-i}\right) & <\int_{\Theta_{-i}} v_{i}\left(\mathbf{x}\left(a_{i}^{\prime}, \theta_{-i}\right), b_{i}^{\prime}, \theta_{-i}\right)-v_{i}\left(\mathbf{x}\left(a_{i}^{\prime}, \theta_{-i}\right), a_{i}^{\prime}, \theta_{-i}\right.
\end{aligned}
$$

This contradicts (7). Therefore (1) follows.

Next take the limit in (6) as $b_{i} \rightarrow a_{i}$, (6) implies that

$$
\frac{\partial \widehat{V}_{i}\left(\theta_{i} ; \mathbf{x}, \mathbf{t}_{i}\right)}{\partial \theta_{i}}=\int_{\Theta_{-i}} \frac{\partial v_{i}\left(\mathbf{x}(\theta), \theta_{i}, \theta_{-i}\right)}{\partial \theta_{i}} d F_{-i}\left(\theta_{-i}\right)
$$

This implies that $\widehat{V}_{i}\left(\theta_{i} ; \mathbf{x}, \mathbf{t}_{i}\right)$ is differentiable in $\left[\underline{\theta}_{i}, \bar{\theta}_{i}\right]$. By assumption 2 and hypothesis (1), $\int_{\Theta_{-i}} \frac{\partial v_{i}\left(\mathbf{x}(\theta), \theta_{i}, \theta_{-i}\right)}{\partial \theta_{i}} d F_{-i}\left(\theta_{-i}\right)$ can only have a countable number of discontinuities, in other words, it is continuous in $\theta_{i}$ almost everywhere with respect to Lebesgue measure. Moreover, $\int_{\Theta_{-i}} \frac{\partial v_{i}\left(\mathbf{x}(\theta), \theta_{i}, \theta_{-i}\right)}{\partial \theta_{i}} d F_{-i}\left(\theta_{-i}\right)$ is bounded on $X \times \Theta$. Hence, by Rudin theorem 6.10. $\frac{\partial \widehat{V}_{i}\left(\theta_{i} ; \mathbf{x}, \mathbf{t}_{i}\right)}{\partial \theta_{i}}$ is Riemann integrable with respect to $\theta_{i} \cdot{ }^{7}$ Then the fundamental theorem of calculus (Rudin 6.21

\footnotetext{
${ }^{7}$ In this case, by Aliprantis abd Border Theorem 11.32, Riemann integrability implies Lebesgue integrability. Riemann integral will coincide with Lebesgue integral.
} 
P134) applies. We have

$$
\widehat{V}_{i}\left(b_{i} ; \mathbf{x}, \mathbf{t}_{i}\right)-\widehat{V}_{i}\left(a_{i} ; \mathbf{x}, \mathbf{t}_{i}\right)=\int_{a_{i}}^{b_{i}} \int_{\Theta_{-i}} \frac{\partial v_{i}\left(\mathbf{x}(\theta), \theta_{i}, \theta_{-i}\right)}{\partial \theta_{i}} d F_{-i}\left(\theta_{-i}\right) d \theta_{i}, \forall a_{i}<b_{i} \in \Theta_{i},
$$

Thus, (2) follows.

Lastly, (3) directly follows from IR condition.

$" \Longrightarrow$ "First since $\frac{\partial v_{i}(x, \theta)}{\partial \theta_{i}} \geq 0,(2)$ and (3) imply $\widehat{V}_{i}\left(\theta_{i} ; \mathbf{x}, \mathbf{t}_{i}\right) \geq r_{i}, \forall \theta_{i} \in \Theta_{i}$. So IR condition holds. Note that BIC condition is equivalent to (4) indeed. Now we need to show that (4) holds.

Suppose (4) does not hold. Then, for some $j, b_{j}, a_{j}$,

$$
\begin{gathered}
\int_{\Theta_{-j}} v_{j}\left(\mathbf{x}\left(a_{j}, \theta_{-j}\right), b_{j}, \theta_{-j}\right) d F_{-j}\left(\theta_{-j}\right)-\int_{\Theta_{-j}} v_{j}\left(\mathbf{x}\left(a_{j}, \theta_{-j}\right), a_{j}, \theta_{-j}\right) d F_{-j}\left(\theta_{-j}\right) \\
<\widehat{V}_{j}\left(b_{j} ; \mathbf{x}, \mathbf{t}_{j}\right)-\widehat{V}_{j}\left(a_{j} ; \mathbf{x}, \mathbf{t}_{j}\right)=\int_{a_{j}}^{b_{j}} \int_{\Theta_{-j}} \frac{\partial v_{j}\left(\mathbf{x}(\theta), \theta_{j}, \theta_{-j}\right)}{\partial \theta_{j}} d F_{-j}\left(\theta_{-j}\right) d \theta_{j}
\end{gathered}
$$

Then

$$
\begin{aligned}
& \int_{a_{j}}^{b_{j}} \int_{\Theta_{-j}} \frac{\partial v_{j}\left(\mathbf{x}(\theta), \theta_{j}, \theta_{-j}\right)}{\partial \theta_{j}} d F_{-j}\left(\theta_{-j}\right) d \theta_{j} \\
< & \int_{\Theta_{-j}} v_{j}\left(\mathbf{x}\left(a_{j}, \theta_{-j}\right), b_{j}, \theta_{-j}\right) d F_{-j}\left(\theta_{-j}\right)-\int_{\Theta_{-j}} v_{j}\left(\mathbf{x}\left(a_{j}, \theta_{-j}\right), a_{j}, \theta_{-j}\right) d F_{-j}\left(\theta_{-j}\right) \\
= & \int_{\Theta_{-j}}\left[v_{j}\left(\mathbf{x}\left(a_{j}, \theta_{-j}\right), b_{j}, \theta_{-j}\right)-v_{j}\left(\mathbf{x}\left(a_{j}, \theta_{-j}\right), a_{j}, \theta_{-j}\right)\right] d F_{-j}\left(\theta_{-j}\right) \\
= & \int_{\Theta_{-j}} \int_{a_{j}}^{b_{j}} \frac{\partial v_{j}\left(\mathbf{x}\left(a_{j}, \theta_{-j}\right), \theta_{j}, \theta_{-j}\right)}{\partial \theta_{j}} d \theta_{j} d F_{-j}\left(\theta_{-j}\right) \\
= & \int_{a_{j}}^{b_{j}} \int_{\Theta_{-j}} \frac{\partial v_{j}\left(\mathbf{x}\left(a_{j}, \theta_{-j}\right), \theta_{j}, \theta_{-j}\right)}{\partial \theta_{j}} d F_{-j}\left(\theta_{-j}\right) d \theta_{j}
\end{aligned}
$$

But (1) implies this is not possible. So this contradiction completes the proof.

\subsection{Characterization of EPIC Mechanisms}

Since EPIC mechanisms do not require Bayesian update beliefs in play, it does not matter whether types are independent or not in ex post implementation. 
Proposition 2 Under assumptions 1-3, (x, t) is $\mathbf{E P I C}$ and IR iff for each $i \in \mathcal{N}, \forall \theta_{-i} \in \Theta_{-i}$,

$$
\begin{aligned}
& \frac{\partial v_{i}\left(\mathbf{x}\left(h, \theta_{-i}\right), \theta\right)}{\partial \theta_{i}} \text { is increasing in } h \text { on } \Theta_{i}, \\
\bar{V}_{i}\left(b_{i}, \theta_{-i} ; \mathbf{x}, \mathbf{t}_{i}\right)-\bar{V}_{i}\left(a_{i}, \theta_{-i} ; \mathbf{x}, \mathbf{t}_{i}\right)= & \int_{a_{i}}^{b_{i}} \frac{\partial v_{i}(\mathbf{x}(\theta), \theta)}{\partial \theta_{i}} d \theta_{i}, \forall a_{i}, b_{i} \in \Theta_{i},{ }^{8} \\
\bar{V}_{i}\left(\underline{\theta}_{i}, \theta_{-i} ; \mathbf{x}, \mathbf{t}_{i}\right) \geq & r_{i} .
\end{aligned}
$$

Proof. " $\Longrightarrow " E P I C$ condition implies that $\forall a_{i}<b_{i} \in \Theta_{i}, \forall \theta_{-i} \in \Theta_{-i}$,

$$
\begin{aligned}
\bar{V}_{i}\left(b_{i}, \theta_{-i} ; \mathbf{x}, \mathbf{t}_{i}\right) \geq & v_{i}\left(\mathbf{x}\left(a_{i}, \theta_{-i}\right), b_{i}, \theta_{-i}\right)+\mathbf{t}_{i}\left(a_{i}, \theta_{-i}\right) \\
= & v_{i}\left(\mathbf{x}\left(a_{i}, \theta_{-i}\right), a_{i}, \theta_{-i}\right)+\mathbf{t}_{i}\left(a_{i}, \theta_{-i}\right) \\
& +v_{i}\left(\mathbf{x}\left(a_{i}, \theta_{-i}\right), b_{i}, \theta_{-i}\right)-v_{i}\left(\mathbf{x}\left(a_{i}, \theta_{-i}\right), a_{i}, \theta_{-i}\right) \\
= & \bar{V}_{i}\left(a_{i}, \theta_{-i} ; \mathbf{x}, \mathbf{t}_{i}\right) \\
& +v_{i}\left(\mathbf{x}\left(a_{i}, \theta_{-i}\right), b_{i}, \theta_{-i}\right)-v_{i}\left(\mathbf{x}\left(a_{i}, \theta_{-i}\right), a_{i}, \theta_{-i}\right)
\end{aligned}
$$

Thus,

$$
\begin{aligned}
& \bar{V}_{i}\left(b_{i}, \theta_{-i} ; \mathbf{x}, \mathbf{t}_{i}\right)-\bar{V}_{i}\left(a_{i}, \theta_{-i} ; \mathbf{x}, \mathbf{t}_{i}\right) \\
\geq & v_{i}\left(\mathbf{x}\left(a_{i}, \theta_{-i}\right), b_{i}, \theta_{-i}\right)-v_{i}\left(\mathbf{x}\left(a_{i}, \theta_{-i}\right), a_{i}, \theta_{-i}\right)
\end{aligned}
$$

Reversing $b_{i}$ and $a_{i}$ and combining (), we have

$$
\begin{aligned}
& v_{i}\left(\mathbf{x}\left(a_{i}, \theta_{-i}\right), b_{i}, \theta_{-i}\right)-v_{i}\left(\mathbf{x}\left(a_{i}, \theta_{-i}\right), a_{i}, \theta_{-i}\right) \\
\leq & \bar{V}_{i}\left(b_{i}, \theta_{-i} ; \mathbf{x}, \mathbf{t}_{i}\right)-\bar{V}_{i}\left(a_{i}, \theta_{-i} ; \mathbf{x}, \mathbf{t}_{i}\right) \\
\leq & v_{i}\left(\mathbf{x}\left(b_{i}, \theta_{-i}\right), b_{i}, \theta_{-i}\right)-v_{i}\left(\mathbf{x}\left(b_{i}, \theta_{-i}\right), a_{i}, \theta_{-i}\right)
\end{aligned}
$$

Thus,

$$
\begin{array}{r}
v_{i}\left(\mathbf{x}\left(a_{i}, \theta_{-i}\right), b_{i}, \theta_{-i}\right)-v_{i}\left(\mathbf{x}\left(a_{i}, \theta_{-i}\right), a_{i}, \theta_{-i}\right) \\
\leq \quad v_{i}\left(\mathbf{x}\left(b_{i}, \theta_{-i}\right), b_{i}, \theta_{-i}\right)-v_{i}\left(\mathbf{x}\left(b_{i}, \theta_{-i}\right), a_{i}, \theta_{-i}\right)
\end{array}
$$

Now suppose (9) does not hold, then $\forall \theta_{-i} \in \Theta_{-i}$,for some $b_{i}^{\prime}>a_{i}^{\prime}$

$$
\frac{\partial v_{i}\left(\mathbf{x}\left(b_{i}^{\prime}, \theta\right), \theta_{i}, \theta_{-i}\right)}{\partial \theta_{i}}<\frac{\partial v_{i}\left(\mathbf{x}\left(a_{i}^{\prime}, \theta\right), \theta_{i}, \theta_{-i}\right)}{\partial \theta_{i}}
$$


Thus,

$$
\begin{aligned}
\int_{a_{i}^{\prime}}^{b_{i}^{\prime}} \frac{\partial v_{i}\left(\mathbf{x}\left(b_{i}^{\prime}, \theta\right), \theta_{i}, \theta_{-i}\right)}{\partial \theta_{i}} d \theta_{i} & <\int_{a_{i}^{\prime}}^{b_{i}^{\prime}} \frac{\partial v_{i}\left(\mathbf{x}\left(a_{i}^{\prime}, \theta\right), \theta_{i}, \theta_{-i}\right)}{\partial \theta_{i}} d \theta_{i} \\
v_{i}\left(\mathbf{x}\left(b_{i}^{\prime}, \theta_{-i}\right), b_{i}^{\prime}, \theta_{-i}\right)-v_{i}\left(\mathbf{x}\left(b_{i}^{\prime}, \theta_{-i}\right), a_{i}^{\prime}, \theta_{-i}\right) & <v_{i}\left(\mathbf{x}\left(a_{i}^{\prime}, \theta_{-i}\right), b_{i}^{\prime}, \theta_{-i}\right)-v_{i}\left(\mathbf{x}\left(a_{i}^{\prime}, \theta_{-i}\right), a_{i}^{\prime}, \theta_{-i}\right)
\end{aligned}
$$

This contradicts (14). Therefore (9) follows.

Next take the limit in (13) as $b_{i} \rightarrow a_{i}$, it implies that

$$
\frac{\partial \bar{V}_{i}\left(\theta_{i}, \theta_{-i} ; \mathbf{x}, \mathbf{t}_{i}\right)}{\partial \theta_{i}}=\frac{\partial v_{i}\left(\mathbf{x}(\theta), \theta_{i}, \theta_{-i}\right)}{\partial \theta_{i}}
$$

This implies that $\bar{V}_{i}\left(\theta_{i} ; \mathbf{x}, \mathbf{t}_{i}\right)$ is differentiable in $\left[\underline{\theta}_{i}, \bar{\theta}_{i}\right]$. Then by similar argument in the proof of Proposition 1,

$$
\bar{V}_{i}\left(b_{i}, \theta_{-i} ; \mathbf{x}, \mathbf{t}_{i}\right)-\bar{V}_{i}\left(a_{i}, \theta_{-i} ; \mathbf{x}, \mathbf{t}_{i}\right)=\int_{a_{i}}^{b_{i}} \frac{\partial v_{i}\left(\mathbf{x}(\theta), \theta_{i}, \theta_{-i}\right)}{\partial \theta_{i}} d \theta_{i}, \forall a_{i}, b_{i} \in \Theta_{i},
$$

Therefore, (10) is satisfied.

Lastly, (11) directly follows from IR condition.

$" \Longleftarrow "$ First since $\frac{\partial v_{i}(x, \theta)}{\partial \theta_{i}} \geq 0,(10)$ and (11) imply $\bar{V}_{i}\left(\underline{\theta}_{i}, \theta_{-i} ; \mathbf{x}, \mathbf{t}_{j}\right) \geq r_{i}, \forall \theta \in \Theta$. So IR condition holds.

Note that EPIC condition is equivalent to (14) indeed. Now we need to show that (14) holds.

Suppose (14) does not hold. Then, for some $j, b_{j}, a_{j}, \widehat{\theta}_{-j}$,

$$
\begin{aligned}
& v_{j}\left(\mathbf{x}\left(a_{j}, \widehat{\theta}_{-j}\right), b_{j}, \widehat{\theta}_{-j}\right)-v_{j}\left(\mathbf{x}\left(a_{j}, \widehat{\theta}_{-j}\right), a_{j}, \widehat{\theta}_{-j}\right) \\
< & \bar{V}_{j}\left(b_{j}, \widehat{\theta}_{-j} ; \mathbf{x}, \mathbf{t}_{j}\right)-\bar{V}_{j}\left(a_{j}, \widehat{\theta}_{-j} ; \mathbf{x}, \mathbf{t}_{j}\right) \\
= & \int_{a_{j}}^{b_{j}} \frac{\partial v_{j}\left(\mathbf{x}\left(\theta_{j}, \widehat{\theta}_{-j}\right), \theta_{j}, \widehat{\theta}_{-j}\right)}{\partial \theta_{j}} d \theta_{j}
\end{aligned}
$$

Thus,

$$
\begin{aligned}
& \int_{a_{j}}^{b_{j}} \frac{\partial v_{j}\left(\mathbf{x}(\theta), \theta_{j}, \widehat{\theta}_{-j}\right)}{\partial \theta_{j}} d \theta_{j} \\
< & v_{j}\left(\mathbf{x}\left(a_{j}, \widehat{\theta}_{-j}\right), b_{j}, \widehat{\theta}_{-j}\right)-v_{j}\left(\mathbf{x}\left(a_{j}, \widehat{\theta}_{-j}\right), a_{j}, \widehat{\theta}_{-j}\right) \\
= & \int_{a_{j}}^{b_{j}} \frac{\partial v_{j}\left(\mathbf{x}\left(a_{j}, \widehat{\theta}_{-j}\right), \theta_{j}, \widehat{\theta}_{-j}\right)}{\partial \theta_{j}} d \theta_{j}
\end{aligned}
$$

But $\frac{\partial v_{j}(x, \theta)}{\partial \theta_{j}} \geq 0$, and (9) implies this is not possible. So this contradiction completes the proof. 


\subsection{Interpretation of Characterization Conditions}

Condition 1 (respectively, 9) is a monotonicity condition. It shows that in the BIC (respectively, EPIC) mechanism, the assignment rule must be such that each $\mathrm{A}_{i}$ 's interim expected (respectively, ex post) marginal payoff from assignments with respect to $\mathrm{A}_{i}$ 's type will increase as $\mathrm{A}_{i}$ 's type report increases for the assignment rule. This is a constraint solely on the assignment rules.

Condition 2 (respectively, 10) is an integration condition. It shows that any difference between $\mathrm{A}_{i}$ 's interim (respectively, ex post) equilibrium payoffs causing by different $\mathrm{A}_{i}$ 's types will be equal to the integral of interim expected marginal payoff (respectively, marginal payoff) from assignments with respect to $\mathrm{A}_{i}$ 's type within the two types.

Condition 2 (respectively, 10) actually corresponds to the envelope theorem as mentioned in Milgrom and Segal (2002). Note that Lebesgue measure is invariant in translation on a closed interval in a Euclidean space. Clearly, condition 2 is equivalent to

$$
\widehat{V}_{i}\left(\theta_{i} ; \mathbf{x}, \mathbf{t}_{i}\right)=\widehat{V}_{i}\left(\underline{\theta}_{i} ; \mathbf{x}, \mathbf{t}_{i}\right)+\int_{\underline{\theta}_{i}}^{\theta_{i}} \int_{\Theta_{-i}} \frac{\partial v_{i}(\mathbf{x}(\theta), \theta)}{\partial \theta_{i}} d F_{-i}\left(\theta_{-i}\right) d \theta_{i}
$$

for each $\theta_{i} \in \Theta_{i}$. Condition 10 is equivalent to

$$
\bar{V}_{i}\left(\theta_{i}, \theta_{-i} ; \mathbf{x}, \mathbf{t}_{i}\right)=\bar{V}_{i}\left(\underline{\theta}_{i}, \theta_{-i} ; \mathbf{x}, \mathbf{t}_{i}\right)+\int_{\underline{\theta}_{i}}^{\theta_{i}} \frac{\partial v_{i}(\mathbf{x}(\theta), \theta)}{\partial \theta_{i}} d \theta_{i}
$$

for each $\theta \in \Theta$.Hence, under any BIC (respectively, EPIC) mechanism, we can know $\mathrm{A}_{i}$ 's Bayesian (respectively ex post) equilibrium payoff mapping with respect to his own type serving as the parameter.

Moreover, equation (7) (respectively (15)), as the corresponding differentiation condition, uncovers how $\mathrm{A}_{i}$ 's Bayesian (respectively ex post) equilibrium payoff will change with respect to his own type.

It is worth noting that condition 3 (respectively 11) is only for IR condition. If we only characterize BIC mechanisms, we can omit condition 3 (respectively 11). Condition 3 (respectively 11), joint with the other two conditions, simplifies the original IR condition. It is only a IR condition for each agent with the lowest type. As long as each agent with the lowest type must be willing to participate, each agent with any other types will always participate.

\subsection{Recoverability of Transfer Rules}

Proposition 3 Under assumptions 1 and 2, condition (2) is equivalent to

$$
\begin{aligned}
\int_{\Theta_{-i}} \mathbf{t}_{i}\left(\theta_{i}, \theta_{-i}\right) d F_{-i}\left(\theta_{-i}\right)= & \int_{\Theta_{-i}}\left[\int_{\underline{\theta}_{i}}^{\theta_{i}} \frac{\partial v_{i}\left(\mathbf{x}\left(h, \theta_{-i}\right), h, \theta_{-i}\right)}{\partial \theta_{i}} d h-v_{i}\left(\mathbf{x}\left(\theta_{i}, \theta_{-i}\right), \theta\right)\right. \\
& \left.+v_{i}\left(\mathbf{x}\left(\underline{\theta}_{i}, \theta_{-i}\right), \underline{\theta}_{i}, \theta_{-i}\right)+\mathbf{t}_{i}\left(\underline{\theta}_{i}, \theta_{-i}\right)\right] d F_{-i}\left(\theta_{-i}\right) .
\end{aligned}
$$


Proof. Given BIC (x,t),

$$
\begin{aligned}
\widehat{V}_{i}\left(\theta_{i} ; \mathbf{x}, \mathbf{t}_{i}\right)-\widehat{V}_{i}\left(\underline{\theta}_{i} ; \mathbf{x}, \mathbf{t}_{i}\right)= & \int_{\underline{\theta}_{i}}^{\theta_{i}} \int_{\Theta_{-i}} \frac{\partial v_{i}\left(\mathbf{x}\left(h, \theta_{-i}\right), h, \theta_{-i}\right)}{\partial \theta_{i}} d F_{-i}\left(\theta_{-i}\right) d h \\
\Longrightarrow & \int_{\Theta_{-i}}\left[v_{i}\left(\mathbf{x}\left(\theta_{i}, \theta_{-i}\right), \theta\right)+\mathbf{t}_{i}\left(\theta_{i}, \theta_{-i}\right)\right] d F_{-i}\left(\theta_{-i}\right) \\
= & \int_{\Theta_{-i}}\left[v_{i}\left(\mathbf{x}\left(\underline{\theta}_{i}, \theta_{-i}\right), \underline{\theta}_{i}, \theta_{-i}\right)+\mathbf{t}_{i}\left(\underline{\theta}_{i}, \theta_{-i}\right)\right] d F_{-i}\left(\theta_{-i}\right) \\
& +\int_{\underline{\theta}_{i}}^{\theta_{i}} \int_{\Theta_{-i}} \frac{\partial v_{i}\left(\mathbf{x}\left(h, \theta_{-i}\right), h, \theta_{-i}\right)}{\partial \theta_{i}} d F_{-i}\left(\theta_{-i}\right) d h \\
\Longrightarrow & \int_{\Theta_{-i}}^{\mathbf{t}_{i}\left(\theta_{i}, \theta_{-i}\right) d F_{-i}\left(\theta_{-i}\right)} \\
= & \int_{\Theta_{-i}}\left[\int_{\underline{\theta}_{i}}^{\theta_{i}} \frac{\partial v_{i}\left(\mathbf{x}\left(h, \theta_{-i}\right), h, \theta_{-i}\right)}{\partial \theta_{i}} d h-v_{i}\left(\mathbf{x}\left(\theta_{i}, \theta_{-i}\right), \theta\right)\right. \\
& \left.+v_{i}\left(\mathbf{x}\left(\underline{\theta}_{i}, \theta_{-i}\right), \underline{\theta}_{i}, \theta_{-i}\right)+\mathbf{t}_{i}\left(\underline{\theta}_{i}, \theta \theta_{-i}\right)\right] d F_{-i}\left(\theta_{-i}\right) .
\end{aligned}
$$

Also note that Lebesgue measure is invariant in translation on a closed interval. So condition (2) is equivalent to (16).

This proposition suggests that in BIC implementation transfer rules can be recovered from assignment rules. If mechanism $(\mathbf{x}, \mathbf{t})$ is $\mathrm{BIC}$, let $\varepsilon\left(\theta_{-i}\right)=v_{i}\left(\mathbf{x}\left(\underline{\theta}_{i}, \theta_{-i}\right), \underline{\theta}_{i}, \theta_{-i}\right)+\mathbf{t}_{i}\left(\underline{\theta}_{i}, \theta_{-i}\right)$. Then,

$$
\int_{\Theta_{-i}} \mathbf{t}_{i}(\theta) d F_{-i}\left(\theta_{-i}\right)=\int_{\Theta_{-i}}\left[\int_{\underline{\theta}_{i}}^{\theta_{i}} \frac{\partial v_{i}\left(\mathbf{x}\left(h, \theta_{-i}\right), h, \theta_{-i}\right)}{\partial \theta_{i}} d h-v_{i}\left(\mathbf{x}\left(\theta_{i}, \theta_{-i}\right), \theta\right)+\varepsilon\left(\theta_{-i}\right)\right] d F_{-i}\left(\theta_{-i}\right)
$$

for some real-valued function $\varepsilon\left(\theta_{-i}\right)$.

Proposition 4 Under assumptions 1 and 2, condition (10) is equivalent to

$$
\mathbf{t}_{i}\left(\theta_{i}, \theta_{-i}\right)=\int_{\underline{\theta}_{i}}^{\theta_{i}} \frac{\partial v_{i}\left(\mathbf{x}\left(h, \theta_{-i}\right), h, \theta_{-i}\right)}{\partial \theta_{i}} d h-v_{i}\left(\mathbf{x}\left(\theta_{i}, \theta_{-i}\right), \theta\right)+v_{i}\left(\mathbf{x}\left(\underline{\theta}_{i}, \theta_{-i}\right), \underline{\theta}_{i}, \theta_{-i}\right)+\mathbf{t}_{i}\left(\underline{\theta}_{i}, \theta_{-i}\right) .
$$

Proof. By condition (10)

$$
\begin{aligned}
\bar{V}_{i}\left(\theta_{i}, \theta_{-i} ; \mathbf{x}, \mathbf{t}_{i}\right)-\bar{V}_{i}\left(\underline{\theta}_{i}, \theta_{-i} ; \mathbf{x}, \mathbf{t}_{i}\right)= & \int_{\underline{\theta}_{i}}^{\theta_{i}} \frac{\partial v_{i}\left(\mathbf{x}\left(h, \theta_{-i}\right), h, \theta_{-i}\right)}{\partial \theta_{i}} d h \\
v_{i}\left(\mathbf{x}\left(\theta_{i}, \theta_{-i}\right), \theta\right)+\mathbf{t}_{i}\left(\theta_{i}, \theta_{-i}\right)= & v_{i}\left(\mathbf{x}\left(\underline{\theta}_{i}, \theta_{-i}\right), \underline{\theta}_{i}, \theta_{-i}\right)+\mathbf{t}_{i}\left(\underline{\theta}_{i}, \theta_{-i}\right)+\int_{\underline{\theta}_{i}}^{\theta_{i}} \frac{\partial v_{i}\left(\mathbf{x}\left(h, \theta_{-i}\right), h, \theta_{-i}\right)}{\partial \theta_{i}} d h \\
\mathbf{t}_{i}\left(\theta_{i}, \theta_{-i}\right)= & \int_{\underline{\theta}_{i}}^{\theta_{i}} \frac{\partial v_{i}\left(\mathbf{x}\left(h, \theta_{-i}\right), h, \theta_{-i}\right)}{\partial \theta_{i}} d h-v_{i}\left(\mathbf{x}\left(\theta_{i}, \theta_{-i}\right), \theta\right) \\
& +v_{i}\left(\mathbf{x}\left(\underline{\theta}_{i}, \theta_{-i}\right), \underline{\theta}_{i}, \theta_{-i}\right)+\mathbf{t}_{i}\left(\underline{\theta}_{i}, \theta_{-i}\right) .
\end{aligned}
$$


Also note that Lebesgue measure is invariant in translation on a closed interval. So condition (10) is equivalent to (17).

This proposition suggests that in BIC implementation transfer rules can also be recovered from assignment rules. Let $\eta\left(\theta_{-i}\right)=v_{i}\left(\mathbf{x}\left(\underline{\theta}_{i}, \theta_{-i}\right), \underline{\theta}_{i}, \theta_{-i}\right)+\mathbf{t}_{i}\left(\underline{\theta}_{i}, \theta_{-i}\right)$. If a mechanism $(\mathbf{x}, \mathbf{t})$ is EPIC, then

$$
\mathbf{t}_{i}\left(\theta_{i}, \theta_{-i}\right)=\int_{\underline{\theta}_{i}}^{\theta_{i}} \frac{\partial v_{i}\left(\mathbf{x}\left(h, \theta_{-i}\right), h, \theta_{-i}\right)}{\partial \theta_{i}} d h-v_{i}\left(\mathbf{x}\left(\theta_{i}, \theta_{-i}\right), \theta\right)+\eta\left(\theta_{-i}\right)
$$

for some real-valued function $\eta\left(\theta_{-i}\right)$.

\subsection{Characterization of Optimal Mechanisms}

Based on characterization results of incentive compatible mechanisms, we can further characterize the optimal incentive compatible mechanisms.

\subsubsection{Characterization of Optimal BIC Mechanisms}

Proposition 5 Suppose that there are no substantive Bayesian update beliefs. Under assumptions 1-3, $\left(\mathbf{x}^{*}, \mathbf{t}^{*}\right)$ is the optimal mechanism solving P1 if

$$
\begin{aligned}
\left(\mathbf{x}^{*}, \mathbf{t}^{*}\right) \in & \arg \max _{\mathbf{x}, \mathbf{t}} \int_{\Theta}\left\{u(\mathbf{x}(\theta), \theta)+\sum_{i=1}^{n}\left(v_{i}(\mathbf{x}(\theta), \theta)\right.\right. \\
& \left.\left.-\frac{1-F_{i}\left(\theta_{i}\right)}{f_{i}\left(\theta_{i}\right)} \frac{\partial v_{i}\left(\mathbf{x}(\theta), \theta_{i}, \theta_{-i}\right)}{\partial \theta_{i}}\right)-\sum_{i=1}^{n} \widehat{V}_{i}\left(\underline{\theta}_{i} ; \mathbf{x}, \mathbf{t}_{i}\right)\right\} d F(\theta) \\
& \text { s.t. } \int_{\Theta_{-i}} \frac{\partial v_{i}\left(\mathbf{x}\left(h, \theta_{-i}\right), \theta_{i}, \theta_{-i}\right)}{\partial \theta_{i}} d F_{-i}\left(\theta_{-i}\right) \text { is increasing in } h \text { on } \Theta_{i}, \\
\widehat{V}_{i}\left(b_{i} ; \mathbf{x}, \mathbf{t}_{i}\right)-\widehat{V}_{i}\left(a_{i} ; \mathbf{x}, \mathbf{t}_{i}\right)= & \int_{a_{i}}^{b_{i}} \int_{\Theta_{-i}}\left[\frac{\partial v_{i}(\mathbf{x}(\theta), \theta)}{\partial \theta_{i}}\right] d F_{-i}\left(\theta_{-i}\right) d \theta_{i}, \forall a_{i}, b_{i} \in \Theta_{i}, \\
\widehat{V}_{i}\left(\underline{\theta}_{i} ; \mathbf{x}, \mathbf{t}_{i}\right) \geq & r_{i}, \\
\text { and } \forall \theta \geq & \Theta, \mathbf{x}(\theta) \in X, \mathbf{t}(\theta) \in T
\end{aligned}
$$

Proof. We can rewrite PL's objective function as

$$
\begin{aligned}
\widehat{U}(\mathbf{x}, \mathbf{t}) & =\int_{\Theta} u(\mathbf{x}(\theta), \theta)-\sum_{i=1}^{n} \mathbf{t}_{i}(\theta) d F(\theta) \\
& =\int_{\Theta} u(\mathbf{x}(\theta), \theta) d F(\theta)+\int_{\Theta} \sum_{i=1}^{n} v_{i}(\mathbf{x}(\theta), \theta) d F(\theta)-\int_{\Theta} \sum_{i=1}^{n} v_{i}(\mathbf{x}(\theta), \theta)+\mathbf{t}_{i}(\theta) d F(\theta)
\end{aligned}
$$


By proposition 1,

$$
\begin{aligned}
& \int_{\Theta} v_{i}(\mathbf{x}(\theta), \theta)+\mathbf{t}_{i}(\theta) d F(\theta) \\
= & \int_{\Theta_{i}} \widehat{V}_{i}\left(\theta_{i} ; \mathbf{x}, \mathbf{t}_{i}\right) d F_{i}\left(\theta_{i}\right) \\
= & \int_{\Theta_{i}}\left[\widehat{V}_{i}\left(\underline{\theta}_{i} ; \mathbf{x}, \mathbf{t}_{i}\right)+\int_{\underline{\theta}_{i}}^{\theta_{i}} \frac{\partial \widehat{V}_{i}\left(s_{i} ; \mathbf{x}, \mathbf{t}_{i}\right)}{\partial \theta_{i}} d s_{i}\right] d F_{i}\left(\theta_{i}\right) \\
= & \widehat{V}_{i}\left(\underline{\theta}_{i} ; \mathbf{x}, \mathbf{t}_{i}\right)+\int_{\Theta_{i}} \int_{\underline{\theta}_{i}}^{\theta_{i}} \frac{\partial \widehat{V}_{i}\left(s_{i} ; \mathbf{x}, \mathbf{t}_{i}\right)}{\partial \theta_{i}} d s_{i} f_{i}\left(\theta_{i}\right) d \theta_{i} \\
= & \left.\widehat{V}_{i}\left(\underline{\theta}_{i} ; \mathbf{x}, \mathbf{t}_{i}\right)+\int_{\Theta_{i}} \int_{s_{i}}^{\bar{\theta}_{i}} \frac{\partial \widehat{V}_{i}\left(s_{i} ; \mathbf{x}, \mathbf{t}_{i}\right)}{\partial \theta_{i}} f_{i}\left(\theta_{i}\right) d \theta_{i} d s_{i} \quad \text { interchange the order of integral. }\right) \\
= & \widehat{V}_{i}\left(\underline{\theta}_{i} ; \mathbf{x}, \mathbf{t}_{i}\right)+\int_{\Theta_{i}}\left(1-F_{i}\left(s_{i}\right)\right) \frac{\partial \widehat{V}_{i}\left(s_{i} ; \mathbf{x}, \mathbf{t}_{i}\right)}{\partial \theta_{i}} d s_{i} \\
= & \widehat{V}_{i}\left(\underline{\theta}_{i} ; \mathbf{x}, \mathbf{t}_{i}\right)+\int_{\Theta_{i}}\left(1-F_{i}\left(s_{i}\right)\right) \int_{\Theta-i} \frac{\partial v_{i}\left(\mathbf{x}\left(s_{i}, \theta_{-i}\right), s_{i}, \theta-i\right)}{\partial \theta_{i}} d F_{-i}\left(\theta_{-i}\right) d s_{i} \\
= & \widehat{V}_{i}\left(\underline{\theta}_{i} ; \mathbf{x}, \mathbf{t}_{i}\right)+\int_{\Theta}\left(1-F_{i}\left(\theta_{i}\right)\right) \frac{\partial v_{i}\left(\mathbf{x}(\theta), \theta_{i}, \theta_{-i}\right)}{\partial \theta_{i}} f_{-i}\left(\theta_{-i}\right) d \theta \\
= & \widehat{V}_{i}\left(\underline{\theta}_{i} ; \mathbf{x}, \mathbf{t}_{i}\right)+\int_{\Theta} \frac{1-F_{i}\left(\theta_{i}\right)}{f_{i}\left(\theta_{i}\right)} \frac{\partial v_{i}\left(\mathbf{x}(\theta), \theta_{i}, \theta_{-i}\right)}{\partial \theta_{i}} f(\theta) d \theta
\end{aligned}
$$

So,

$\widehat{U}(\mathbf{x}, \mathbf{t})=\int_{\Theta}\left\{u(\mathbf{x}(\theta), \theta)+\sum_{i=1}^{n}\left(v_{i}(\mathbf{x}(\theta), \theta)-\frac{1-F_{i}\left(\theta_{i}\right)}{f_{i}\left(\theta_{i}\right)} \frac{\partial v_{i}\left(\mathbf{x}(\theta), \theta_{i}, \theta_{-i}\right)}{\partial \theta_{i}}\right)-\sum_{i=1}^{n} \widehat{V}_{i}\left(\underline{\theta}_{i} ; \mathbf{x}, \mathbf{t}_{i}\right)\right\} d F(\theta)$

Thus, P1 is equivalent to an alternative problem $\mathbf{P} \mathbf{1}^{\prime}$ that maximizes (19) subject to (1), (2), (3) and (18).

Based on Proposition 3, we can construct an optimal mechanism in which $\mathbf{x}^{*}$ can be independently determined and then $\mathbf{t}^{*}$ is subsequently determined by $\mathbf{x}^{*}$. Moreover, the optimal objective value of PL can be uniquely determined by $\mathbf{x}^{*}$ independent of $\mathbf{t}^{*}$.

Corollary 1 Suppose that there are no substantive Bayesian update beliefs. Under assumptions 
1-3, suppose that

$$
\begin{aligned}
\mathbf{x}^{*} \in & \arg \max _{\mathbf{x}} \int_{\Theta} u(\mathbf{x}(\theta), \theta)+\sum_{i=1}^{n}\left(v_{i}(\mathbf{x}(\theta), \theta)\right. \\
& \left.-\frac{1-F_{i}\left(\theta_{i}\right)}{f_{i}\left(\theta_{i}\right)} \frac{\partial v_{i}\left(\mathbf{x}(\theta), \theta_{i}, \theta_{-i}\right)}{\partial \theta_{i}}\right) d F(\theta) \\
& \text { s.t. } \int_{\Theta_{-i}} \frac{\partial v_{i}\left(\mathbf{x}\left(h, \theta_{-i}\right), \theta_{i}, \theta_{-i}\right)}{\partial \theta_{i}} d F_{-i}\left(\theta_{-i}\right) \text { is increasing in } h \text { on } \Theta_{i} \\
\forall \theta \in & \Theta, \mathbf{x}(\theta) \in X . \\
\text { In addition, } \forall i \in & \mathcal{N}, \theta \in \Theta, \\
\mathbf{t}_{i}^{*}(\theta) \text { is such that } \int_{\Theta_{-i}} \mathbf{t}_{i}^{*}(\theta) d F_{-i}\left(\theta_{-i}\right)= & \int_{\Theta_{-i}}\left[\int_{\underline{\theta}_{i}}^{\theta_{i}} \frac{\partial v_{i}\left(\mathbf{x}^{*}\left(s_{i}, \theta_{-i}\right), s_{i}, \theta_{-i}\right)}{\partial \theta_{i}} d s_{i}-v_{i}\left(\mathbf{x}^{*}(\theta), \theta\right)\right] d F_{-i}\left(\theta_{-i}\right)+r_{i}, \\
\text { as long as } \mathbf{t}(\theta) \in & T, \forall \theta \in \Theta, \\
\mathbf{t}_{i}(\theta) d F_{-i}\left(\theta_{-i}\right)= & \int_{\Theta_{-i}}\left[\int_{\underline{\theta}_{i}}^{\theta_{i}} \frac{\partial v_{i}\left(\mathbf{x}\left(s_{i}, \theta_{-i}\right), s_{i}, \theta_{-i}\right)}{\partial \theta_{i}} d s_{i}-v_{i}(\mathbf{x}(\theta), \theta)\right] d F_{-i}\left(\theta_{-i}\right)+r_{i} .
\end{aligned}
$$

Then such a combination $\left(\mathbf{x}^{*}, \mathbf{t}^{*}\right)$ is the optimal mechanism solving P1.

Moreover, the optimal objective value of $P L$ in $\boldsymbol{P} \mathbf{1}$ is always equal to

$$
\int_{\Theta} u\left(\mathbf{x}^{*}(\theta), \theta\right)+\sum_{i=1}^{n}\left(v_{i}\left(\mathbf{x}^{*}(\theta), \theta\right)-\frac{1-F_{i}\left(\theta_{i}\right)}{f_{i}\left(\theta_{i}\right)} \frac{\partial v_{i}\left(\mathbf{x}^{*}(\theta), \theta_{i}, \theta_{-i}\right)}{\partial \theta_{i}}\right) d F(\theta)-\sum_{i=1}^{n} r_{i} .
$$

Proof. (2) and (3) give

$$
\begin{aligned}
r_{i} & \leq \widehat{V}_{i}\left(\underline{\theta}_{i} ; \mathbf{x}, \mathbf{t}_{i}\right)=\widehat{V}_{i}\left(\theta_{i} ; \mathbf{x}, \mathbf{t}_{i}\right)-\int_{\underline{\theta}_{i}}^{\theta_{i}} \int_{\Theta_{-i}} \frac{\partial v_{i}\left(\mathbf{x}\left(s_{i}, \theta_{-i}\right), s_{i}, \theta_{-i}\right)}{\partial \theta_{i}} d F_{-i}\left(\theta_{-i}\right) d s_{i} \\
& =\int_{\Theta_{-i}} v_{i}(\mathbf{x}(\theta), \theta)+\mathbf{t}_{i}(\theta) d F_{-i}\left(\theta_{-i}\right)-\int_{\underline{\theta}_{i}}^{\theta_{i}} \int_{\Theta_{-i}} \frac{\partial v_{i}\left(\mathbf{x}\left(s_{i}, \theta_{-i}\right), s_{i}, \theta_{-i}\right)}{\partial \theta_{i}} d F_{-i}\left(\theta_{-i}\right) d s_{i} \\
& =\int_{\Theta_{-i}} v_{i}(\mathbf{x}(\theta), \theta)+\mathbf{t}_{i}(\theta) d F_{-i}\left(\theta_{-i}\right)-\int_{\Theta_{-i}} \int_{\underline{\theta}_{i}}^{\theta_{i}} \frac{\partial v_{i}\left(\mathbf{x}\left(s_{i}, \theta_{-i}\right), s_{i}, \theta_{-i}\right)}{\partial \theta_{i}} d s_{i} d F_{-i}\left(\theta_{-i}\right) \\
& =\int_{\Theta_{-i}}\left\{v_{i}(\mathbf{x}(\theta), \theta)+\mathbf{t}_{i}(\theta)-\int_{\underline{\theta}_{i}}^{\theta_{i}} \frac{\partial v_{i}\left(\mathbf{x}\left(s_{i}, \theta_{-i}\right), s_{i}, \theta_{-i}\right)}{\partial \theta_{i}} d s_{i}\right\} d F_{-i}\left(\theta_{-i}\right)
\end{aligned}
$$

Thus, if PL can pick $\mathbf{t}_{i}$ such that

$$
\int_{\Theta_{-i}} \mathbf{t}_{i}(\theta) d F_{-i}\left(\theta_{-i}\right)=\int_{\Theta_{-i}}\left[\int_{\underline{\theta}_{i}}^{\theta_{i}} \frac{\partial v_{i}\left(\mathbf{x}\left(s_{i}, \theta_{-i}\right), s_{i}, \theta_{-i}\right)}{\partial \theta_{i}} d s_{i}-v_{i}(\mathbf{x}(\theta), \theta)\right] d F_{-i}\left(\theta_{-i}\right)+r_{i}
$$

for each $i$ as long as $\mathbf{t}(\theta) \in T$, she satisfies both (2) and (3), and she gets $\sum_{i=1}^{n} \widehat{V}_{i}\left(\underline{\theta}_{i} ; \mathbf{x}, \mathbf{t}_{i}\right)=\sum_{i=1}^{n} r_{i}$, which is the best possible value for this term in (19). Therefore, we can entirely drop t from 
$\mathbf{P} 1^{\prime}$. Moreover, the objective function (19) in $\mathbf{P} \mathbf{1}^{\prime}$ can be simplified to

$$
\int_{\Theta} u(\mathbf{x}(\theta), \theta)+\sum_{i=1}^{n}\left(v_{i}(\mathbf{x}(\theta), \theta)-\frac{1-F_{i}\left(\theta_{i}\right)}{f_{i}\left(\theta_{i}\right)} \frac{\partial v_{i}\left(\mathbf{x}(\theta), \theta_{i}, \theta_{-i}\right)}{\partial \theta_{i}}\right) d F(\theta)
$$

and (1) and (18) are the only constraints left. This completes the proof.

\subsubsection{Characterization of Optimal EPIC Mechanisms}

Proposition 6 Under assumptions 1-3, $\left(\mathrm{x}^{*}, \mathbf{t}^{*}\right)$ is the optimal mechanism solving P2 iff

$$
\begin{aligned}
\left(\mathbf{x}^{*}, \mathbf{t}^{*}\right) \in & \arg \max _{\mathbf{x}, \mathbf{t}} \int_{\Theta}\{u(\mathbf{x}(\theta), \theta) \\
& \left.+\sum_{i=1}^{n}\left(v_{i}(\mathbf{x}(\theta), \theta)-\frac{1-F_{i}\left(\theta_{i}\right)}{f_{i}\left(\theta_{i}\right)} \frac{\partial v_{i}\left(\mathbf{x}\left(\theta_{i}, \theta_{-i}\right), \theta_{i}, \theta_{-i}\right)}{\partial \theta_{i}}\right)\right\} d F(\theta) \\
& -\sum_{i=1}^{n} \int_{\Theta} \bar{V}_{i}\left(\underline{\theta}_{i}, \theta_{-i} ; \mathbf{x}, \mathbf{t}_{i}\right) d F(\theta) \\
\text { s.t. } \forall \theta_{-i} \in & \Theta_{-i}, \frac{\partial v_{i}\left(\mathbf{x}\left(h, \theta_{-i}\right), \theta_{i}, \theta_{-i}\right)}{\partial \theta_{i}} \text { is increasing in } h \text { on } \Theta_{i}, \\
\bar{V}_{i}\left(b_{i}, \theta_{-i} ; \mathbf{x}, \mathbf{t}_{i}\right)-\bar{V}_{i}\left(a_{i}, \theta_{-i} ; \mathbf{x}, \mathbf{t}_{i}\right)= & \int_{\left[a_{i}, b_{i}\right]} \frac{\partial v_{i}(\mathbf{x}(\theta), \theta)}{\partial \theta_{i}} d \theta_{i}, \forall a_{i}, b_{i} \in \Theta_{i}, \\
\bar{V}_{i}\left(\underline{\theta}_{i}, \theta_{-i} ; \mathbf{x}, \mathbf{t}_{i}\right) \geq & r_{i}, \\
\text { and } \forall \theta \in & \Theta, \mathbf{x}(\theta) \in X, \mathbf{t}(\theta) \in T
\end{aligned}
$$

Proof. We can rewrite PL's objective function as

$$
\begin{aligned}
\widehat{U}(\mathbf{x}, \mathbf{t}) & =\int_{\Theta} u(\mathbf{x}(\theta), \theta)-\sum_{i=1}^{n} \mathbf{t}_{i}(\theta) d F(\theta) \\
& =\int_{\Theta} u(\mathbf{x}(\theta), \theta) d F(\theta)+\int_{\Theta} \sum_{i=1}^{n} v_{i}(\mathbf{x}(\theta), \theta) d F(\theta)-\int_{\Theta} \sum_{i=1}^{n} v_{i}(\mathbf{x}(\theta), \theta)+\mathbf{t}_{i}(\theta) d F(\theta)
\end{aligned}
$$


By proposition 3,

$$
\begin{aligned}
& \int_{\Theta} v_{i}(\mathbf{x}(\theta), \theta)+\mathbf{t}_{i}(\theta) d F(\theta) \\
= & \int_{\Theta} \bar{V}_{i}\left(\theta_{i}, \theta_{-i} ; \mathbf{x}, \mathbf{t}_{i}\right) d F(\theta) \\
= & \int_{\Theta} \bar{V}_{i}\left(\underline{\theta}_{i}, \theta_{-i} ; \mathbf{x}, \mathbf{t}_{i}\right) d F(\theta)+\int_{\Theta} \int_{\underline{\theta}_{i}}^{\theta_{i}} \frac{\partial \bar{V}_{i}\left(s_{i}, \theta_{-i} ; \mathbf{x}, \mathbf{t}_{i}\right)}{\partial \theta_{i}} d s_{i} d F(\theta) \\
= & \int_{\Theta} \bar{V}_{i}\left(\underline{\theta}_{i}, \theta_{-i} ; \mathbf{x}, \mathbf{t}_{i}\right) d F(\theta)+\int_{\Theta_{i}} \int_{\Theta_{-i}} \int_{\underline{\theta}_{i}}^{\theta_{i}} \frac{\partial \bar{V}_{i}\left(s_{i}, \theta_{-i} ; \mathbf{x}, \mathbf{t}_{i}\right)}{\partial \theta_{i}} d s_{i} d F_{-i}\left(\theta_{-i} \mid \theta_{i}\right) d F_{i}\left(\theta_{i}\right) \\
= & \int_{\Theta} \bar{V}_{i}\left(\underline{\theta}_{i}, \theta_{-i} ; \mathbf{x}, \mathbf{t}_{i}\right) d F(\theta)+\int_{\Theta_{i}} \int_{\underline{\theta}_{i}}^{\theta_{i}}\left(\int_{\Theta_{-i}} \frac{\partial \bar{V}_{i}\left(s_{i}, \theta_{-i} ; \mathbf{x}, \mathbf{t}_{i}\right)}{\partial \theta_{i}} d F_{-i}\left(\theta_{-i} \mid s_{i}\right)\right) d s_{i} f_{i}\left(\theta_{i}\right) d \theta_{i} \\
= & \int_{\Theta} \bar{V}_{i}\left(\underline{\theta}_{i}, \theta_{-i} ; \mathbf{x}, \mathbf{t}_{i}\right) d F(\theta)+\int_{\Theta_{i}} \int_{s_{i}}^{\bar{\theta}_{i}}\left(\int_{\Theta_{-i}} \frac{\partial \bar{V}_{i}\left(s_{i}, \theta \theta_{-i} ; \mathbf{x}, \mathbf{t}_{i}\right)}{\partial \theta_{i}} d F_{-i}\left(\theta_{-i} \mid \theta_{i}\right)\right) f_{i}\left(\theta_{i}\right) d \theta_{i} d s_{i} \\
= & \int_{\Theta} \bar{V}_{i}\left(\underline{\theta}_{i}, \theta_{-i} ; \mathbf{x}, \mathbf{t}_{i}\right) d F(\theta)+\int_{\Theta_{i}}\left(1-F_{i}\left(s_{i}\right)\right) \int_{\Theta_{-i}} \frac{\partial v_{i}\left(\mathbf{x}\left(s_{i}, \theta_{-i}\right), s_{i}, \theta_{-i}\right)}{\partial \theta_{i}} d F_{-i}\left(\theta_{-i} \mid s_{i}\right) d s_{i} \\
= & \int_{\Theta} \bar{V}_{i}\left(\underline{\theta}_{i}, \theta_{-i} ; \mathbf{x}, \mathbf{t}_{i}\right) d F(\theta)+\int_{\Theta_{i}} \int_{\Theta_{-i}}\left(1-F_{i}\left(\theta_{i}\right)\right) \frac{\partial v_{i}\left(\mathbf{x}\left(\theta_{i}, \theta_{-i}\right), \theta_{i}, \theta_{-i}\right)}{\partial \theta_{i}} d F_{-i}\left(\theta_{-i} \mid \theta_{i}\right) d \theta_{i} \\
= & \int_{\Theta} \bar{V}_{i}\left(\underline{\theta}_{i}, \theta_{-i} ; \mathbf{x}, \mathbf{t}_{i}\right) d F(\theta)+\int_{\Theta}\left(1-F_{i}\left(\theta_{i}\right)\right) \frac{\partial v_{i}\left(\mathbf{x}\left(\theta_{i}, \theta_{-i}\right), \theta_{i}, \theta_{-i}\right)}{\partial \theta_{i}} f_{-i}\left(\theta_{-i} \mid \theta_{i}\right) d \theta \\
= & \int_{\Theta} \bar{V}_{i}\left(\underline{\theta}_{i}, \theta_{-i} ; \mathbf{x}, \mathbf{t}_{i}\right) d F(\theta)+\int_{\Theta} \frac{1-F_{i}\left(\theta_{i}\right)}{f_{i}\left(\theta_{i}\right)} \frac{\partial v_{i}\left(\mathbf{x}\left(\theta_{i}, \theta_{-i}\right), \theta_{i}, \theta \theta_{-i}\right)}{\partial \theta_{i}} f(\theta) d \theta
\end{aligned}
$$

So,

$$
\begin{aligned}
\widehat{U}(\mathbf{x}, \mathbf{t})=\int_{\Theta}\{u(\mathbf{x}(\theta), \theta) & +\sum_{i=1}^{n}\left(v_{i}(\mathbf{x}(\theta), \theta)-\frac{1-F_{i}\left(\theta_{i}\right)}{f_{i}\left(\theta_{i}\right)} \frac{\partial v_{i}\left(\mathbf{x}\left(\theta_{i}, \theta_{-i}\right), \theta_{i}, \theta_{-i}\right)}{\partial \theta_{i}}\right\} f(\theta) d \theta \\
& -\sum_{i=1}^{n} \int_{\Theta} \bar{V}_{i}\left(\underline{\theta}_{i}, \theta_{-i} ; \mathbf{x}, \mathbf{t}_{i}\right) d F(\theta)
\end{aligned}
$$

Thus, P1 is equivalent to an alternative problem $\mathbf{P} 1^{\prime}$ maximizing (22) subject to (9), (10), (11) and (18).

Based on Proposition 4, we can construct an optimal mechanism in which $\mathbf{x}^{*}$ can be independently determined and then $\mathbf{t}^{*}$ is subsequently determined by $\mathbf{x}^{*}$. Moreover, the optimal objective value of PL is uniquely determined by $\mathbf{x}^{*}$ independent of $\mathbf{t}^{*}$. 
Corollary 2 Under assumptions 1-3, if there exists

$$
\begin{aligned}
\mathbf{x}^{*} \in & \arg \max _{\mathbf{x}} \int_{\Theta}\{u(\mathbf{x}(\theta), \theta) \\
& \left.+\sum_{i=1}^{n}\left(v_{i}(\mathbf{x}(\theta), \theta)-\frac{1-F_{i}\left(\theta_{i}\right)}{f_{i}\left(\theta_{i}\right)} \frac{\partial v_{i}\left(\mathbf{x}\left(\theta_{i}, \theta_{-i}\right), \theta_{i}, \theta_{-i}\right)}{\partial \theta_{i}}\right)\right\} d F(\theta) \\
\text { s.t. } \forall \theta_{-i} \in & \Theta_{-i}, \frac{\partial v_{i}\left(\mathbf{x}\left(h, \theta_{-i}\right), \theta_{i}, \theta_{-i}\right)}{\partial \theta_{i}} \text { is increasing in } h \text { on } \Theta_{i}, \\
\forall \theta \in & \Theta, \mathbf{x}(\theta) \in X . \\
\text { and if } \forall i \in & \mathcal{N}, \theta \in \Theta, \\
\mathbf{t}_{i}^{*}(\theta)= & \int_{\underline{\theta}_{i}}^{\theta_{i}} \frac{\partial v_{i}\left(\mathbf{x}^{*}\left(s_{i}, \theta_{-i}\right), s_{i}, \theta_{-i}\right)}{\partial \theta_{i}} d s_{i}-v_{i}\left(\mathbf{x}^{*}(\theta), \theta\right)+r_{i},
\end{aligned}
$$

as long as $\mathbf{t}(\theta) \in T, \forall \theta \in \Theta$,

$$
\text { where } \mathbf{t}_{i}(\theta)=\int_{\underline{\theta}_{i}}^{\theta_{i}} \frac{\partial v_{i}\left(\mathbf{x}\left(s_{i}, \theta_{-i}\right), s_{i}, \theta_{-i}\right)}{\partial \theta_{i}} d s_{i}-v_{i}(\mathbf{x}(\theta), \theta)+r_{i}
$$

then such a combination $\left(\mathbf{x}^{*}, \mathbf{t}^{*}\right)$ is the optimal mechanism solving P2.

Moreover, the optimal objective value of $P L$ in $\boldsymbol{P} \mathscr{2}$ is always equal to

$$
\int_{\Theta}\left\{u\left(\mathbf{x}^{*}(\theta), \theta\right)+\sum_{i=1}^{n}\left(v_{i}\left(\mathbf{x}^{*}(\theta), \theta\right)-\frac{1-F_{i}\left(\theta_{i}\right)}{f_{i}\left(\theta_{i}\right)} \frac{\partial v_{i}\left(\mathbf{x}^{*}(\theta), \theta_{i}, \theta_{-i}\right)}{\partial \theta_{i}}\right)\right\} d F(\theta)-\sum_{i=1}^{n} r_{i} .
$$

Proof. $\forall \theta_{-i} \in \Theta_{-i},(15)$ and (16) give

$$
\begin{aligned}
r_{i} & \leq \bar{V}_{i}\left(\underline{\theta}_{i}, \theta_{-i} ; \mathbf{x}, \mathbf{t}_{i}\right)=\bar{V}_{i}\left(\theta_{i}, \theta_{-i} ; \mathbf{x}, \mathbf{t}_{i}\right)-\int_{\underline{\theta}_{i}}^{\theta_{i}} \frac{\partial v_{i}\left(\mathbf{x}\left(s_{i}, \theta_{-i}\right), s_{i}, \theta_{-i}\right)}{\partial \theta_{i}} d s_{i} \\
& =v_{i}(\mathbf{x}(\theta), \theta)+\mathbf{t}_{i}(\theta)-\int_{\underline{\theta}_{i}}^{\theta_{i}} \frac{\partial v_{i}\left(\mathbf{x}\left(s_{i}, \theta_{-i}\right), s_{i}, \theta_{-i}\right)}{\partial \theta_{i}} d s_{i} \\
& =v_{i}(\mathbf{x}(\theta), \theta)+\mathbf{t}_{i}(\theta)-\int_{\underline{\theta}_{i}}^{\theta_{i}} \frac{\partial v_{i}\left(\mathbf{x}\left(s_{i}, \theta_{-i}\right), s_{i}, \theta_{-i}\right)}{\partial \theta_{i}} d s_{i} \\
& =v_{i}(\mathbf{x}(\theta), \theta)+\mathbf{t}_{i}(\theta)-\int_{\underline{\theta}_{i}}^{\theta_{i}} \frac{\partial v_{i}\left(\mathbf{x}\left(s_{i}, \theta_{-i}\right), s_{i}, \theta_{-i}\right)}{\partial \theta_{i}} d s_{i}
\end{aligned}
$$

Thus, if PL can pick

$$
\mathbf{t}_{i}(\theta)=\int_{\underline{\theta}_{i}}^{\theta_{i}} \frac{\partial v_{i}\left(\mathbf{x}\left(s_{i}, \theta_{-i}\right), s_{i}, \theta_{-i}\right)}{\partial \theta_{i}} d s_{i}-v_{i}(\mathbf{x}(\theta), \theta)+r_{i}
$$

for each $i$ as long as $\mathbf{t}(\theta) \in T$, she satisfies both (10) and (11), and she gets

$$
\sum_{i=1}^{n} \int_{\Theta} \bar{V}_{i}\left(\underline{\theta}_{i}, \theta_{-i} ; \mathbf{x}, \mathbf{t}_{i}\right) d F(\theta)=\sum_{i=1}^{n} r_{i}
$$


which is the best possible value for this term in (22). Therefore, we can entirely drop t from $\mathbf{P} \mathbf{1}^{\prime}$. Moreover, the objective function (22) in $\mathbf{P} \mathbf{1}^{\prime}$ can be simplified to

$$
\int_{\Theta} u(\mathbf{x}(\theta), \theta)+\sum_{i=1}^{n}\left(v_{i}(\mathbf{x}(\theta), \theta)-\frac{1-F_{i}\left(\theta_{i}\right)}{f_{i}\left(\theta_{i}\right)} \frac{\partial v_{i}\left(\mathbf{x}(\theta), \theta_{i}, \theta_{-i}\right)}{\partial \theta_{i}}\right) d F(\theta)
$$

and (9) and (18) are the only constraints left. This completes the proof.

\subsubsection{Welfare Analysis}

Corollary 1 implies that in optimum the total expected surplus, equal to total expected benefit (because the total transfer cancels out), has been distorted by an amount equal to the total expected information rents of the agents plus the welfare loss from outside options (reservation

utilities). In details, the total expected surplus is $\int_{\Theta} u(\mathbf{x}(\theta), \theta)+\sum_{i=1}^{n}\left(v_{i}(\mathbf{x}(\theta), \theta) d F(\theta)\right.$. The total expected information rents of the agents is $\left.\int_{\Theta} \sum_{i=1}^{n} \frac{1-F_{i}\left(\theta_{i}\right)}{f_{i}\left(\theta_{i}\right)} \frac{\partial v_{i}\left(\mathbf{x}(\theta), \theta_{i}, \theta_{-i}\right)}{\partial \theta_{i}}\right) d F(\theta)$. The welfare loss from outside options is $\sum_{i=1}^{n} r_{i}$. In this case, (21) represents the virtual surplus after distortion of information rents and the welfare loss from outside options, which represents the second best situation.

The total expected information rents reflects how much the private information (adverse selection) will impair social welfare. It is clear that this distortion will positively related to the number of informed agents, the inverse of hazard rate of $\theta_{i}, \frac{1-F_{i}\left(\theta_{i}\right)}{f_{i}\left(\theta_{i}\right)}$, and $\mathrm{A}_{i}$ 's marginal utility of $\theta_{i}, \frac{\partial v_{i}(x, \theta)}{\partial \theta_{i}}$. Each $\frac{1-F_{i}\left(\theta_{i}\right)}{f_{i}\left(\theta_{i}\right)} \frac{\partial v_{i}\left(\mathbf{x}(\theta), \theta_{i}, \theta_{-i}\right)}{\partial \theta_{i}}$ can also be viewed as $\mathrm{A}_{i}$ 's marginal utility of $\theta_{i}$ discounted by hazard rate in overall expectation.

Corollary 2 shares the similar economic intuition as Corollary 1 . The only difference is that there is a stricter constraint on the feasible assignment rules for ex post implementation than Bayesian implementation.

\subsection{Discussions}

\subsubsection{The Role of Assumption 4}

In addition, if strict increasing difference (assumption 4) holds, we can simplify the condition (4) as the monotonicity properties on assignment functions directly. It leads to a more direct characterization of the EPIC mechanisms per se.

Lemma 1 Under Assumption 4, if $\forall b_{i}>a_{i}$ in $\Theta_{i}, \forall \theta_{-i} \in \Theta_{-i}$,

$$
v_{i}\left(x^{\prime \prime}, b_{i}, \theta_{-i}\right)-v_{i}\left(x^{\prime}, b_{i}, \theta_{-i}\right) \geq v_{i}\left(x^{\prime \prime}, a_{i}, \theta_{-i}\right)-v_{i}\left(x^{\prime}, a_{i}, \theta_{-i}\right),
$$

then $x^{\prime \prime} \geq x^{\prime}$ in $\mathcal{X}$. 
Proof. Suppose that $x^{\prime \prime}<x^{\prime}$. By Assumption 4,

$$
v_{i}\left(x^{\prime \prime}, b_{i}, \theta_{-i}\right)-v_{i}\left(x^{\prime}, b_{i}, \theta_{-i}\right)<v_{i}\left(x^{\prime \prime}, a_{i}, \theta_{-i}\right)-v_{i}\left(x^{\prime}, a_{i}, \theta_{-i}\right)
$$

It obviously reaches a contradiction.

Corollary 3 Under assumption 4, condition (9) is equivalent to (9') $\mathbf{x}\left(h, \theta_{-i}\right)$ is increasing in $h$ on $\Theta_{i}$.

Proof. " $\Longleftarrow "$ Assumption 3 implies that $\frac{\partial v_{i}\left(x, \theta_{i}, \theta_{-i}\right)}{\partial \theta_{i}}$ is increasing in $x$. Then the rest of the proof is straightforward.

$" \Longrightarrow " \forall b_{i}>a_{i}$ in $\Theta_{i}, \forall \theta_{-i} \in \Theta_{-i}$,

$$
\int_{a_{i}}^{b_{i}} \frac{\partial v_{i}\left(\mathbf{x}\left(b_{i}, \theta_{-i}\right), \theta\right)}{\partial \theta_{i}} d \theta_{i} \geq \int_{a_{i}}^{b_{i}} \frac{\partial v_{i}\left(\mathbf{x}\left(a_{i}, \theta_{-i}\right), \theta\right)}{\partial \theta_{i}} d \theta_{i}
$$

Hence,

$$
v_{i}\left(\mathbf{x}\left(b_{i}, \theta_{-i}\right), b_{i}, \theta_{-i}\right)-v_{i}\left(\mathbf{x}\left(b_{i}, \theta_{-i}\right), a_{i}, \theta_{-i}\right) \geq v_{i}\left(\mathbf{x}\left(a_{i}, \theta_{-i}\right), b_{i}, \theta_{-i}\right)-v_{i}\left(\mathbf{x}\left(a_{i}, \theta_{-i}\right), a_{i}, \theta_{-i}\right)
$$

Furthermore,

$$
v_{i}\left(\mathbf{x}\left(b_{i}, \theta_{-i}\right), b_{i}, \theta_{-i}\right)-v_{i}\left(\mathbf{x}\left(a_{i}, \theta_{-i}\right), b_{i}, \theta_{-i}\right) \geq v_{i}\left(\mathbf{x}\left(b_{i}, \theta_{-i}\right), a_{i}, \theta_{-i}\right)-v_{i}\left(\mathbf{x}\left(a_{i}, \theta_{-i}\right), a_{i}, \theta_{-i}\right)
$$

By Lemma 1, we can conclude that $\mathbf{x}\left(h, \theta_{-i}\right)$ is increasing in $h$ on $\Theta_{i}$.

\subsubsection{Separable Assignment Rules}

Now we consider a particular situation in which the assignment rules can be separable across the agents. For each $i \in \mathcal{N}$, suppose $\mathcal{X}_{i} \subseteq \mathbb{R}$. Let $\mathcal{X}=\prod_{i \in \mathcal{N}} \mathcal{X}_{i}$. Let $\mathbf{x}(\theta)=\prod_{i \in \mathcal{N}} \mathbf{x}_{i}(\theta)$, where each $\mathbf{x}_{i}$ is a Borel measurable mapping from $\Theta$ to $\mathcal{X}_{i}$. The agents may have no externalities in assignments, that is, each $v_{i}(x, \theta) \equiv v_{i}\left(x_{i}, \theta\right)$. In this case, we will have a little revision on corollary 3.

Corollary 4 If the agents have no externalities in assignments, condition (9) is equivalent to $\left(9^{\prime \prime}\right) \mathbf{x}_{i}\left(h, \theta_{-i}\right)$ is increasing in $h$ on $\Theta_{i}$, for each $i$.

$\left(9^{\prime \prime}\right)$ is actually weaker than $\left(9^{\prime}\right)$. We also need to replace $\left(9^{\prime}\right)$ with $\left(9^{\prime \prime}\right)$ in all the results concerning EPIC mechanisms accordingly. The rest of the results aforementioned will still hold.

\subsubsection{Private Valuations and Dominant Strategy Implementation}

If the agents have private valuations, that is, each $v_{i}(x, \theta) \equiv v_{i}\left(x, \theta_{i}\right)$, ex post implementation will degenerate to dominant strategy implementation. In this case, clearly, all the results aforementioned will still hold, but will appear in accordingly refined forms. 


\section{Applications}

\subsection{Comparison between EPIC and BIC Mechanisms}

The most remarkable application of our characterization results is comparison and equivalence between EPIC and BIC mechanisms.

\subsubsection{Equivalence between EPIC and BIC Mechanisms}

Clearly, if the types are allowed to be correlated, whenever a direct mechanism is EPIC (and IR), it must be BIC (and IR). Furthermore, by propositions 1 and 2, if a direct mechanism is EPIC (and IR), it also must be a BIC (and IR) mechanism with no substantive Bayesian update beliefs. But the converse is not true at large.

Now we consider an interim payoff-equivalence between mechanisms.

Definition 2 A mechanism (x, t) is said to be interim payoff-equivalent to a mechanism $\left(\mathbf{x}^{\prime}, \mathbf{t}^{\prime}\right)$ if for all $i \in \mathcal{N}$ and $\theta_{i} \in \Theta_{i}$,

$$
\begin{aligned}
\mathbf{x}(\theta) & =\mathbf{x}^{\prime}(\theta), \\
\text { and } \int_{\Theta_{-i}} \mathbf{t}_{i}(\theta) d F_{-i}\left(\theta_{-i} \mid \theta_{i}\right) & =\int_{\Theta_{-i}} \mathbf{t}_{i}^{\prime}(\theta) d F_{-i}\left(\theta_{-i} \mid \theta_{i}\right),
\end{aligned}
$$

Clearly, in such interim payoff equivalence, for a given mechanism $(\mathbf{x}, \mathbf{t})$, the other mechanism $\left(\mathbf{x}^{\prime}, \mathbf{t}^{\prime}\right)$ can deliver same expected payoff to each agent. Moreover, such interim payoff equivalence may bring analytical advantage. Obviously, it can preserve BIC and IR properties.

Proposition 7 Under assumptions 1-3, for any BIC (and IR) mechanism (x, t) with no substantive Bayesian update beliefs, if $\frac{\partial v_{i}\left(\mathbf{x}\left(h, \theta_{-i}\right), \theta_{i}, \theta_{-i}\right)}{\partial \theta_{i}}$ is increasing in $h$ on $\Theta_{i},\left(\right.$ and $\bar{V}_{i}\left(\underline{\theta}_{i}, \theta_{-i} ; \mathbf{x}, \mathbf{t}_{i}\right) \geq$ $r_{i}$, ) then there exists an interim-payoff-equivalent EPIC (and IR) mechanism $(\mathbf{x}, \widetilde{\mathbf{t}})$.

Proof. For any BIC mechanism $(\mathbf{x}, \mathbf{t})$, by proposition $3,(\mathbf{x}, \mathbf{t})$ must satisfy

$\int_{\Theta_{-i}} \mathbf{t}_{i}\left(\theta_{i}, \theta_{-i}\right) d F_{-i}\left(\theta_{-i}\right)=\int_{\Theta_{-i}}\left[\int_{\underline{\theta}_{i}}^{\theta_{i}} \frac{\partial v_{i}\left(\mathbf{x}\left(h, \theta_{-i}\right), h, \theta_{-i}\right)}{\partial \theta_{i}} d h-v_{i}\left(\mathbf{x}\left(\theta_{i}, \theta_{-i}\right), \theta\right)+\varepsilon\left(\theta_{-i}\right)\right] d F_{-i}\left(\theta_{-i}\right)$,

where $\varepsilon\left(\theta_{-i}\right)=v_{i}\left(\mathbf{x}\left(\underline{\theta}_{i}, \theta_{-i}\right), \underline{\theta}_{i}, \theta_{-i}\right)+\mathbf{t}_{i}\left(\underline{\theta}_{i}, \theta_{-i}\right)$.

We need to show that there exists an EPIC mechanism $(\mathbf{x}, \widetilde{\mathbf{t}})$ satisfying

$$
\begin{aligned}
\mathbf{x}(\theta) & =\mathbf{x}^{\prime}(\theta) \\
\text { and } \int_{\Theta_{-i}} \mathbf{t}_{i}(\theta) d F_{-i}\left(\theta_{-i}\right) & =\int_{\Theta_{-i}}^{\prime} \widetilde{\mathbf{t}}_{i}(\theta) d F_{-i}\left(\theta_{-i}\right) .
\end{aligned}
$$


First by proposition 4 , any mechanism $\left(\mathbf{x}, \mathbf{t}^{\prime}\right)$ satisfying

$$
\mathbf{t}_{i}^{\prime}\left(\theta_{i}, \theta_{-i}\right)=\int_{\underline{\theta}_{i}}^{\theta_{i}} \frac{\partial v_{i}\left(\mathbf{x}\left(h, \theta_{-i}\right), h, \theta_{-i}\right)}{\partial \theta_{i}} d h-v_{i}\left(\mathbf{x}\left(\theta_{i}, \theta_{-i}\right), \theta\right)+\eta\left(\theta_{-i}\right),
$$

where $\eta\left(\theta_{-i}\right)=v_{i}\left(\mathbf{x}\left(\underline{\theta}_{i}, \theta_{-i}\right), \underline{\theta}_{i}, \theta_{-i}\right)+\mathbf{t}_{i}^{\prime}\left(\underline{\theta}_{i}, \theta_{-i}\right)$, must be EPIC, since it satisfies conditions () and () .

Now define $\widetilde{\mathbf{t}}_{i}\left(\theta_{i}, \theta_{-i}\right)=\mathbf{t}_{i}^{\prime}\left(\theta_{i}, \theta_{-i}\right)-\eta\left(\theta_{-i}\right)+\varepsilon\left(\theta_{-i}\right)$.

Firstly,

$$
\begin{aligned}
\int_{\Theta_{-i}} \widetilde{\mathbf{t}}_{i}(\theta) d F_{-i}\left(\theta_{-i}\right) & =\int_{\Theta_{-i}}\left[\int_{\underline{\theta}_{i}}^{\theta_{i}} \frac{\partial v_{i}\left(\mathbf{x}\left(h, \theta_{-i}\right), h, \theta_{-i}\right)}{\partial \theta_{i}} d h-v_{i}\left(\mathbf{x}\left(\theta_{i}, \theta_{-i}\right), \theta\right)+\varepsilon\left(\theta_{-i}\right)\right] d F_{-i}\left(\theta_{-i}\right) \\
& =\int_{\Theta_{-i}} \mathbf{t}_{i}(\theta) d F_{-i}\left(\theta_{-i}\right) .
\end{aligned}
$$

Secondly, we need to show $(\mathbf{x}, \widetilde{\mathbf{t}})$ is EPIC. We have already know $\frac{\partial v_{i}\left(\mathbf{x}\left(h, \theta_{-i}\right), \theta_{i}, \theta_{-i}\right)}{\partial \theta_{i}}$ is increasing in $h$ on $\Theta_{i}$. Now it suffices to show

$$
\bar{V}_{i}\left(b_{i}, \theta_{-i} ; \mathbf{x}, \widetilde{\mathbf{t}}_{i}\right)-\bar{V}_{i}\left(a_{i}, \theta_{-i} ; \mathbf{x}, \widetilde{\mathbf{t}}_{i}\right)=\int_{a_{i}}^{b_{i}} \frac{\partial v_{i}(\mathbf{x}(\theta), \theta)}{\partial \theta_{i}} d \theta_{i}, \forall a_{i}, b_{i} \in \Theta_{i},
$$

In fact, for each $i \in \mathcal{N}, \forall \theta_{-i} \in \Theta_{-i}$,

$$
\begin{aligned}
\bar{V}_{i}\left(b_{i}, \theta_{-i} ; \mathbf{x}, \widetilde{\mathbf{t}}_{i}\right)-\bar{V}_{i}\left(a_{i}, \theta_{-i} ; \mathbf{x}, \widetilde{\mathbf{t}}_{i}\right) & =v_{i}\left(\mathbf{x}\left(b_{i}, \theta_{-i}\right), b_{i}, \theta_{-i}\right)+\widetilde{\mathbf{t}}_{i}\left(b_{i}, \theta_{-i}\right)-v_{i}\left(\mathbf{x}\left(a_{i}, \theta_{-i}\right), a_{i}, \theta_{-i}\right)-\widetilde{\mathbf{t}}_{i}\left(a_{i}, \theta_{-i}\right) \\
& =v_{i}\left(\mathbf{x}\left(b_{i}, \theta_{-i}\right), b_{i}, \theta_{-i}\right)+\mathbf{t}_{i}^{\prime}\left(b_{i}, \theta_{-i}\right)-v_{i}\left(\mathbf{x}\left(a_{i}, \theta_{-i}\right), a_{i}, \theta_{-i}\right)-\mathbf{t}_{i}^{\prime}\left(a_{i}, \theta_{-i}\right) \\
& =\bar{V}_{i}\left(b_{i}, \theta_{-i} ; \mathbf{x}, \mathbf{t}_{i}^{\prime}\right)-\bar{V}_{i}\left(a_{i}, \theta_{-i} ; \mathbf{x}, \mathbf{t}_{i}^{\prime}\right) \\
& =\int_{a_{i}}^{b_{i}} \frac{\partial v_{i}(\mathbf{x}(\theta), \theta)}{\partial \theta_{i}} d \theta_{i}, \text { since }\left(\mathbf{x}, \mathbf{t}^{\prime}\right) \text { is EPIC. }
\end{aligned}
$$

Therefore $(\mathbf{x}, \widetilde{\mathbf{t}})$ is EPIC.

As for IR, we know

$$
\begin{aligned}
\bar{V}_{i}\left(\underline{\theta}_{i}, \theta_{-i} ; \mathbf{x}, \widetilde{\mathbf{t}}_{i}\right)= & v_{i}\left(\mathbf{x}\left(\underline{\theta}_{i}, \theta_{-i}\right), \underline{\theta}_{i}, \theta_{-i}\right)+\widetilde{\mathbf{t}}_{i}\left(\underline{\theta}_{i}, \theta_{-i}\right) \\
= & v_{i}\left(\mathbf{x}\left(\underline{\theta}_{i}, \theta_{-i}\right), \underline{\theta}_{i}, \theta_{-i}\right)+\mathbf{t}_{i}^{\prime}\left(\underline{\theta}_{i}, \theta_{-i}\right)-\left[v_{i}\left(\mathbf{x}\left(\underline{\theta}_{i}, \theta_{-i}\right), \underline{\theta}_{i}, \theta_{-i}\right)+\mathbf{t}_{i}^{\prime}\left(\underline{\theta}_{i}, \theta_{-i}\right)\right] \\
& +v_{i}\left(\mathbf{x}\left(\underline{\theta}_{i}, \theta_{-i}\right), \underline{\theta}_{i}, \theta_{-i}\right)+\mathbf{t}_{i}\left(\underline{\theta}_{i}, \theta_{-i}\right) \\
= & v_{i}\left(\mathbf{x}\left(\underline{\theta}_{i}, \theta_{-i}\right), \underline{\theta}_{i}, \theta_{-i}\right)+\mathbf{t}_{i}\left(\underline{\theta}_{i}, \theta_{-i}\right) \\
= & \left.\bar{V}_{i}\left(\underline{\theta}_{i}, \theta_{-i} ; \mathbf{x}, \mathbf{t}_{i}\right) \geq r_{i} . \text { by hypothesis }\right)
\end{aligned}
$$

Therefore $(\mathbf{x}, \widetilde{\mathbf{t}})$ is also IR.

This proposition can be viewed as a generalization of proposition 4 in Mookherjee and Reichelstein (1992), which is only effective in the private valuation context with the one- 
dimensional condensation property.

\subsubsection{Comparison between Optimal EPIC and BIC Mechanisms}

By comparison between corollaries 1 and 2, we can see that optimal EPIC mechanisms will be weakly dominated by optimal BIC mechanisms with no substantive Bayesian update beliefs in quasilinear environments. This indicates that the effect of optimal EPIC mechanism design even cannot exceed that of optimal BIC mechanism design with no substantive Bayesian update beliefs.

In light of Manelli and Vincent's work (2010), in the linear environment with private valuations, we can establish the equivalence of optimal dominant strategy IC (DSIC) mechanisms and optimal BIC mechanism design with no substantive Bayesian update beliefs. Consider a linear environment, as a reduced form of quasi-linear environment, in which each agent's payoff is linear in both assignment rule and transfer rule. In detail, $v_{i}(x, \theta) \equiv \theta_{i} x_{i}$, and

$u(x, \theta) \equiv \sum_{i=1}^{n} z_{i}\left(\theta_{i}\right) x_{i}+c(\theta)$, where $z_{i}: \Theta_{i} \rightarrow \mathbb{R}$ and $c: \Theta \rightarrow \mathbb{R}$ are continuous functions. There are also separable assignment rules $\mathbf{x}(\theta)=\prod_{i \in \mathcal{N}} \mathbf{x}_{i}(\theta)$ satisfying $\forall \theta \in \Theta, \mathbf{x}(\theta) \in X=\left\{x: \sum_{i} x_{i} \leq 1\right.$ and $x_{i} \geq 0$ for each $\left.i\right\}$.

According to Manelli and Vincent (2010), for any BIC mechanism (x,t), there exists a dominant strategy IC mechanism $\left(\mathbf{x}^{\prime}, \mathbf{t}^{\prime}\right)$ that generates the same expected assignment rules, i.e. $\int_{\Theta_{-i}} \mathbf{x}_{-i}(\theta) d F\left(\theta_{-i}\right)=\int_{\Theta_{-i}} \mathbf{x}_{-i}^{\prime}(\theta) d F\left(\theta_{-i}\right)$ almost everywhere for each $i$.

Assumption 4 automatically applies in this linear environment. By corollary 1, if there exists $\left(\mathbf{x}^{*}, \mathbf{t}^{*}\right)$ as below,

$$
\begin{aligned}
\mathbf{x}^{*} \in \arg \max _{\mathbf{x}^{\prime}} \int_{\Theta} \sum_{i=1}^{n} z_{i}\left(\theta_{i}\right) \mathbf{x}_{i}(\theta)+c(\theta)+\sum_{i=1}^{n} \theta_{i} \mathbf{x}_{i}(\theta) & \left.-\frac{1-F_{i}\left(\theta_{i}\right)}{f_{i}\left(\theta_{i}\right)} \mathbf{x}_{i}(\theta)\right) d F(\theta) \\
& \text { s.t. } \mathbf{x}_{i}\left(h, \theta_{-i}\right) \text { is increasing in } h \text { on } \Theta_{i} \text {, for each } i . \\
\forall \theta \in & \Theta, \mathbf{x}(\theta) \in X . \\
\text { and } \forall i \in & \mathcal{N}, \theta \in \Theta, \\
\mathbf{t}_{i}^{*}(\theta) \text { is such that } \int_{\Theta_{-i}} \mathbf{t}_{i}^{*}(\theta) d F_{-i}\left(\theta_{-i}\right)= & \int_{\Theta_{-i}}\left[\int_{\underline{\theta}_{i}}^{\theta_{i}} \frac{\partial v_{i}\left(\mathbf{x}^{*}\left(s_{i}, \theta_{-i}\right), s_{i}, \theta_{-i}\right)}{\partial \theta_{i}} d s_{i}-v_{i}\left(\mathbf{x}^{*}(\theta), \theta\right)\right] d F_{-i}\left(\theta_{-i}\right)+r_{i}, \\
\text { as long as } \mathbf{t}(\theta) \in & T, \forall \theta \in \Theta^{\prime}, \\
\int_{\Theta_{-i}} \mathbf{t}_{i}(\theta) d F_{-i}\left(\theta_{-i}\right)= & \int_{\Theta_{-i}}\left[\int_{\underline{\theta}_{i}}^{\theta_{i}} \frac{\partial v_{i}\left(\mathbf{x}\left(s_{i}, \theta_{-i}\right), s_{i}, \theta_{-i}\right)}{\partial \theta_{i}} d s_{i}-v_{i}(\mathbf{x}(\theta), \theta)\right] d F_{-i}\left(\theta_{-i}\right)+r_{i},
\end{aligned}
$$

then $\left(\mathbf{x}^{*}, \mathbf{t}^{*}\right)$ is the optimal solution to $\mathbf{P} \mathbf{1}$ with no substantive Bayesian update beliefs. 
Note that

$$
\begin{aligned}
& \int_{\Theta} \sum_{i=1}^{n} z_{i}\left(\theta_{i}\right) \mathbf{x}_{i}(\theta)+c(\theta)+\sum_{i=1}^{n}\left(\theta_{i} \mathbf{x}_{i}(\theta)-\frac{1-F_{i}\left(\theta_{i}\right)}{f_{i}\left(\theta_{i}\right)} \mathbf{x}_{i}(\theta)\right) d F(\theta) \\
= & \sum_{i=1}^{n}\left\{\int_{\Theta_{i}} z_{i}\left(\theta_{i}\right)\left[\int_{\Theta_{-i}} \mathbf{x}_{i}(\theta) d F_{-i}\left(\theta_{-i}\right)\right] d F_{i}\left(\theta_{i}\right)\right\}+\int_{\Theta} c(\theta) d F(\theta) \\
& +\sum_{i=1}^{n}\left\{\int_{\Theta_{i}}\left(\theta_{i}-\frac{1-F_{i}\left(\theta_{i}\right)}{f_{i}\left(\theta_{i}\right)}\right)\left[\int_{\Theta_{-i}} \mathbf{x}_{i}(\theta) d F_{-i}\left(\theta_{-i}\right)\right] d F_{i}\left(\theta_{i}\right)\right\} \\
= & \sum_{i=1}^{n}\left\{\int_{\Theta_{i}} z_{i}\left(\theta_{i}\right)\left[\int_{\Theta_{-i}} \mathbf{x}_{i}^{\prime}(\theta) d F_{-i}\left(\theta_{-i}\right)\right] d F_{i}\left(\theta_{i}\right)\right\}+\int_{\Theta} c(\theta) d F(\theta) \\
& +\sum_{i=1}^{n}\left\{\int_{\Theta_{i}}\left(\theta_{i}-\frac{1-F_{i}\left(\theta_{i}\right)}{f_{i}\left(\theta_{i}\right)}\right)\left[\int_{\Theta_{-i}} \mathbf{x}_{i}^{\prime}(\theta) d F_{-i}\left(\theta_{-i}\right)\right] d F_{i}\left(\theta_{i}\right)\right\} \\
= & \int_{\Theta} z_{i}\left(\theta_{i}\right) \mathbf{x}_{i}^{\prime}(\theta)+c(\theta)+\sum_{i=1}^{n}\left(\theta_{i} \mathbf{x}_{i}^{\prime}(\theta)-\frac{1-F_{i}\left(\theta_{i}\right)}{f_{i}\left(\theta_{i}\right)} \mathbf{x}_{i}^{\prime}(\theta)\right) d F(\theta)
\end{aligned}
$$

By Manelli and Vincent (2010), for any BIC mechanism (x,t), there exists a dominant strategy IC mechanism $\left(\mathbf{x}^{\prime}, \mathbf{t}^{\prime}\right)$ that generates the same expected assignment rules, i.e. $\int_{\Theta_{-i}} \mathbf{x}_{-i}(\theta) d F\left(\theta_{-i}\right)=$ $\int_{\Theta_{-i}} \mathbf{x}_{-i}^{\prime}(\theta) d F\left(\theta_{-i}\right)$ almost everywhere for each $i$. Thus there must exist

$$
\begin{aligned}
\mathbf{x}^{* *} \in & \arg \max _{\mathbf{x}^{\prime}} \int_{\Theta} \sum_{i=1}^{n} z_{i}\left(\theta_{i}\right) \mathbf{x}_{i}^{\prime}(\theta)+c(\theta)+\sum_{i=1}^{n} \theta_{i} \mathbf{x}_{i}^{\prime}(\theta) \\
& \left.-\frac{1-F_{i}\left(\theta_{i}\right)}{f_{i}\left(\theta_{i}\right)} \mathbf{x}_{i}^{\prime}(\theta)\right) d F(\theta) \\
& \text { s.t. } \int_{\Theta_{-i}} \mathbf{x}_{i}^{\prime}\left(h, \theta_{-i}\right) d F_{-i}\left(\theta_{-i}\right) \text { is increasing in } h \text { on } \Theta_{i}, \\
\forall \theta \in & \Theta, \mathbf{x}(\theta) \in X .
\end{aligned}
$$

Let

$$
\mathbf{t}_{i}^{* *}(\theta)=\int_{\underline{\theta}_{i}}^{\theta_{i}} \frac{\partial v_{i}\left(\mathbf{x}^{* *}\left(s_{i}, \theta_{-i}\right), s_{i}, \theta_{-i}\right)}{\partial \theta_{i}} d s_{i}-v_{i}\left(\mathbf{x}^{*}(\theta), \theta\right)+r_{i} .
$$

Then $\left(\mathbf{x}^{* *}, \mathbf{t}^{* *}\right)$ is DSIC and solves P2. It will bring to PL the same objective value as $\left(\mathbf{x}^{*}, \mathbf{t}^{*}\right)$ does. The optimal objective value is equal to

$$
\left.\int_{\Theta} \sum_{i=1}^{n} z_{i}\left(\theta_{i}\right) \mathbf{x}_{i}^{*}(\theta)+c(\theta)+\sum_{i=1}^{n}\left(\theta_{i}-\frac{1-F_{i}\left(\theta_{i}\right)}{f_{i}\left(\theta_{i}\right)}\right) \mathbf{x}_{i}^{*}(\theta)\right\} d F(\theta)-\sum_{i=1}^{n} r_{i}
$$

If $u(x, \theta) \equiv \sum_{i=1}^{n} \theta_{i} x_{i}$,both $\left(\mathbf{x}^{*}, \mathbf{t}^{*}\right)$ and $\left(\mathbf{x}^{* *}, \mathbf{t}^{* *}\right)$ are efficient mechanisms with the same optimal objective value (normally social welfare).

This can also reflect how much efficiency will lose if we restrict attention from optimal BIC 
mechanism design to optimal DSIC mechanism design when the types can allow to be correlated in such a linear environment. We can simply transfer the comparison between these two to the comparison between general optimal BIC mechanism design and optimal BIC mechanism design without substantive Bayesian update beliefs.

\subsection{Comparison between Mechanisms and Menus}

In Chen (2012)'s work, characterization of EPIC mechanisms can also help identify the conditions for strategic dominance between centralized mechanism design and decentralized menu design.

A joint-based mechanism is a pair of functions $(\mathbf{x}, \mathbf{t})$ satisfying $\mathbf{x}(\theta) \in X \subseteq \mathcal{X}$ and $\mathbf{t}(\theta) \in T \subseteq \mathbb{R}^{n}$. An individual-based mechanism is a pair of functions $(\overline{\mathbf{x}}, \overline{\mathbf{t}})$ satisfying $\overline{\mathbf{x}}(\theta) \in$ $X \subseteq \mathcal{X}$ and $\overline{\mathbf{t}}(\theta) \in T \subseteq \mathbb{R}^{n}$, where $\overline{\mathbf{x}}(\theta)=\left(\overline{\mathbf{x}}_{1}\left(\theta_{1}\right), \cdots, \overline{\mathbf{x}}_{n}\left(\theta_{n}\right)\right)$ and $\overline{\mathbf{x}}(\theta)=\left(\overline{\mathbf{x}}_{1}\left(\theta_{1}\right), \cdots, \overline{\mathbf{x}}_{n}\left(\theta_{n}\right)\right)$ for each $\theta$. Chen shows that individual-based EPIC mechanism design is strategically equivalent to ex post menu design. Thus, comparison between centralized mechanism design and decentralized menu design gets boiled down to comparison between joint-based EPIC mechanisms and individual-based EPIC mechanisms.

\section{Assumption 5}

$$
V_{i}\left(x_{i}, s_{i}, t\right) \equiv w_{i}(t) x_{i}+s_{i},
$$

where $w_{i}: T \rightarrow \mathbb{R}$ is the payoff function from the agents' assignments. $v_{i}$ is continuous. For all $t, \frac{\partial w_{i}(t)}{\partial t_{i}}>0 . \mathrm{A}_{i}$ has the reservation utility $r_{i} \in \mathbb{R}$.

According to corollary 2, we have such a characterization result for optimal joint-based mechanisms as below.

$$
\begin{aligned}
\mathbf{x}^{*} \in & \arg \max _{\mathbf{x}} \int_{T}\{u(\mathbf{x}(\theta), \theta) \\
& \left.\left.+\sum_{i=1}^{n} w_{i}(\theta) \mathbf{x}_{i}(\theta)-\frac{1-F_{i}\left(\theta_{i}\right)}{f_{i}\left(\theta_{i}\right)} \frac{\partial w_{i}(\theta)}{\partial \theta_{i}} \mathbf{x}_{i}(\theta)\right)\right\} d F(\theta)
\end{aligned}
$$

s.t. for each $\theta_{-i} \in T_{-i}, \mathbf{x}(\theta)$ is increasing in $\theta_{i}$,

$$
\text { for each } \theta \in T, \mathbf{x}(\theta) \in X \text {, }
$$

and for each $i \in \mathcal{N}, \theta \in T$,

$$
\mathbf{t}_{i}^{*}(\theta)=\int_{\underline{\theta}_{i}}^{\theta_{i}} \frac{\partial w_{i}\left(s_{i}, \theta_{-i}\right)}{\partial \theta_{i}} \mathbf{x}^{*}\left(s_{i}, \theta_{-i}\right) d s_{i}-w_{i}(\theta) \mathbf{x}_{i}^{*}(\theta)+r_{i}
$$

as long as $\mathbf{t}(\theta) \in T, \forall \theta \in \Theta$, where

$$
\mathbf{t}_{i}(\theta)=\int_{\underline{\theta}_{i}}^{\theta_{i}} \frac{\partial w_{i}\left(\mathbf{x}\left(s_{i}, \theta_{-i}\right), s_{i}, \theta_{-i}\right)}{\partial \theta_{i}} d s_{i}-w_{i}(\mathbf{x}(\theta), \theta)+r_{i}
$$

Then $\left(\mathbf{x}^{*}, \mathbf{t}^{*}\right)$ solves the optimal joint-based EPIC mechanism design problem. Moreover, 
the optimal objective value of PL is always equal to

$$
\int_{\theta}\left\{u\left(\mathbf{x}^{*}(\theta), \theta\right)+\sum_{i=1}^{n}\left(v_{i}(\theta) \mathbf{x}_{i}^{*}(\theta)-\frac{1-F_{i}\left(\theta_{i}\right)}{f_{i}\left(\theta_{i}\right)} \frac{\partial v_{i}(\theta)}{\partial \theta_{i}} \mathbf{x}^{*}(\theta)\right)\right\} d F(\theta)-\sum_{i=1}^{n} r_{i} .
$$

By a similar argument, we can obtain a corresponding characterization result for optimal individual-based mechanisms as below. If

$$
\begin{aligned}
\overline{\mathbf{x}}^{*} \in & \arg \max _{\overline{\mathbf{x}}} \int_{\theta}\{u(\overline{\mathbf{x}}(\theta), \theta) \\
& \left.+\sum_{i=1}^{n}\left(w_{i}(\theta) \overline{\mathbf{x}}_{i}\left(\theta_{i}\right)-\frac{1-F_{i}\left(\theta_{i}\right)}{f_{i}\left(\theta_{i}\right)} \frac{\partial w_{i}(\theta)}{\partial \theta_{i}} \overline{\mathbf{x}}_{i}(\theta)\right)\right\} d F(\theta) \\
& \text { s.t. } \overline{\mathbf{x}}_{i}\left(\theta_{i}\right) \text { is increasing in } \theta_{i},
\end{aligned}
$$

for each $\theta \in \theta, \overline{\mathbf{x}}(\theta) \in X$,

and for each $i \in \mathcal{N}, \theta \in \theta$,

$$
\overline{\mathbf{t}}_{i}^{*}(\theta)=\int_{\underline{\theta}_{i}}^{\theta_{i}} \frac{\partial w_{i}\left(s_{i}, \theta_{-i}\right)}{\partial \theta_{i}} \overline{\mathbf{x}}_{i}^{*}\left(s_{i}\right) d s_{i}-w_{i}(\theta) \overline{\mathbf{x}}_{i}^{*}\left(\theta_{i}\right)+r_{i},
$$

as long as $\overline{\mathbf{t}}(\theta) \in T, \forall \theta \in \Theta$, where

$$
\overline{\mathbf{t}}_{i}(\theta)=\int_{\underline{\theta}_{i}}^{\theta_{i}} \frac{\partial w_{i}\left(\mathbf{x}\left(s_{i}, \theta_{-i}\right), s_{i}, \theta_{-i}\right)}{\partial \theta_{i}} d s_{i}-w_{i}(\mathbf{x}(\theta), \theta)+r_{i}
$$

Then $\left(\overline{\mathbf{x}}^{*}, \overline{\mathbf{t}}^{*}\right)$ solves the optimal individual-based EPIC mechanism design problem. Moreover, the optimal objective value of PL is always equal to

$$
\int_{\theta}\left\{u\left(\overline{\mathbf{x}}^{*}(\theta), \theta\right)+\sum_{i=1}^{n}\left(w_{i}(\theta) \overline{\mathbf{x}}_{i}^{*}(\theta)-\frac{1-F_{i}\left(\theta_{i}\right)}{f_{i}\left(\theta_{i}\right)} \frac{\partial w_{i}(\theta)}{\partial \theta_{i}} \overline{\mathbf{x}}_{i}^{*}(\theta)\right)\right\} d F(\theta)-\sum_{i=1}^{n} r_{i} .
$$

According to Chen, we have a result for strategic dominance of joint-based EPIC mechanism design as below.

Proposition 8 In the quasilinear environment, under assumptions 1-5, suppose that $X$ is also convex. If

$$
u(x+b, \theta)-u(x, \theta)>\sum_{i=1}^{n} b_{i}\left(\frac{1-F_{i}\left(\theta_{i}\right)}{f_{i}\left(\theta_{i}\right)} \frac{\partial w_{i}(\theta)}{\partial \theta_{i}}-w_{i}(\theta)\right)
$$

for any $\theta, x$, and $b=\prod_{i=1}^{n} b_{i} \in \mathbb{R}^{n}$, joint-based EPIC mechanism design strictly dominates both individual-based EPIC mechanism design and also ex post menu design.

For any type profile, if the change in PL's payoff induced by a change in $x$ should exceed the change in the agents' total information rent net of payoffs from assignments induced by that change in $x$, then joint-based mechanism can make PL strictly better off, because it can lead to greater benefit for PL than the information loss incurred by the agents. 


\subsection{Optimal Surplus Extraction}

In the auction design context, Let $x_{i}$ represent the probability of bidder $i$ 's wining the object and $m_{i}$ represent the payment from the bidder $i$. Assume the real-valued valuation function $h_{i}$ is continuos in $\theta$. Now the designer's objective is to extract the surplus as possible as she can. Let

$$
\begin{aligned}
V_{i}(x, t, \theta) & \equiv w_{i}(\theta) x_{i}-m_{i}, v_{i}(x, \theta) \equiv w_{i}(\theta) x_{i} \\
U(x, t, \theta) & \equiv \sum_{i=1}^{n} m_{i}-\max _{i \in \mathcal{N}} w_{i}(\theta) .
\end{aligned}
$$

Set $u(x, \theta)=-\max _{i \in \mathcal{N}} v_{i}(\theta)$ and $t_{i}=-m_{i}$. Assume that for each $i, \frac{\partial w_{i}(\theta)}{\partial \theta_{i}} \geq 0$ and $r_{i}=0$.

Then in ex post implementation, by our characterization results, if

$$
\begin{aligned}
\mathbf{x}^{*} \in & \arg \max _{\mathbf{x}} \int_{\Theta}\left\{-\max _{i \in \mathcal{N}} h_{i}(\theta)\right. \\
& \left.+\sum_{i=1}^{n}\left(h_{i}(\theta) \mathbf{x}_{i}^{*}(\theta)-\frac{1-F_{i}\left(\theta_{i}\right)}{f_{i}\left(\theta_{i}\right)} \frac{\partial h_{i}\left(\theta_{i}, \theta_{-i}\right)}{\partial \theta_{i}} \mathbf{x}_{i}^{*}(\theta)\right)\right\} d F(\theta) \\
\text { s.t. } \forall \theta_{-i} \in & \Theta_{-i}, \mathbf{x}_{i}(\theta) \text { is increasing in } \theta_{i} \\
\forall \theta \in & \Theta, \sum_{i=1}^{n} \mathbf{x}_{i}(\theta) \leq 1, \mathbf{x}_{i}(\theta) \geq 0
\end{aligned}
$$

then $\mathbf{x}^{*}$ solves $\mathbf{P 2}$ and uniquely determines the optimal surplus extraction. The optimal surplus extraction should be non-positive. It is equal to

$$
\int_{\Theta}\left\{-\max _{i \in \mathcal{N}} h_{i}(\theta)+\sum_{i=1}^{n}\left(h_{i}(\theta) \mathbf{x}_{i}^{*}(\theta)-\frac{1-F_{i}\left(\theta_{i}\right)}{f_{i}\left(\theta_{i}\right)} \frac{\partial h_{i}\left(\theta_{i}, \theta_{-i}\right)}{\partial \theta_{i}} \mathbf{x}_{i}^{*}(\theta)\right)\right\} d F(\theta)
$$

Its best possible value would be zero. In that case, full surplus extraction will appear. It occurs only when $\mathrm{x}^{*}$ satisfies

$$
\left.\int_{\Theta} \max _{i \in \mathcal{N}} h_{i}(\theta) d F(\theta)=\int_{\Theta} \sum_{i=1}^{n}\left(h_{i}(\theta) \mathbf{x}_{i}^{*}(\theta)-\frac{1-F_{i}\left(\theta_{i}\right)}{f_{i}\left(\theta_{i}\right)} \frac{\partial h_{i}\left(\theta_{i}, \theta_{-i}\right)}{\partial \theta_{i}} \mathbf{x}_{i}^{*}(\theta)\right)\right\} d F(\theta)
$$

\section{Conclusion}

Our characterization results make the analysis more tractable in quasilinear environment. It addresses more interdependence features in multi-agency situations. The results covers both BIC and EPIC mechanisms. In particular, we can characterize the optimal incentive compatible mechanisms. It will help simplify or specify the analytical issues in optimal mechanism design problems. 


\section{References}

[1] Ash,R.B., Doleans-Dade, C.A., 1999, Probability \& Measure Theory, Second Edition, Academic Press

[2] Aliprantis, C. D., Border, K. C., 2006, "Infinite Dimensional Analysis: A Hitchhiker's Guide," Springer-Verlag, Berlin-Heidelberg.

[3] Chen, Y., 2013, "Centralization or Decentralization in Multi-agency Contracting Games?" Indiana University CAEPR working paper \#2012-008

[4] Manelli, A. M., Vincent, D. R., 2010, "Bayesian and Dominant-Strategy Implementation in the Independent Private-Values Model," Econometrica, Volume 78 No.6 1905-1938

[5] Milgrom, P., Segal, I., 2002, "Envelope Theorems for Arbitrary Choice Sets," Econometrica, Volume 70 No.2 583-601

[6] Mookherjee, D., Reichelstein, S., 1992, "Dominant-Strategy Implementation of Bayesian Incentive Compatible Allocation Rules," Journal of Economic Theory, Volume 56 378-399

[7] Myerson, R. B., 1981, "Optimal Auction Design," Mathematics of Operations Research, Volume 6 Issue 1, 58-73

[8] Rudin, W., 1976, "Principles of mathematical analysis," Third Edition, Mcgraw-Hill Companies, Inc. 